\title{
The Endophytic Fungus Cyanodermella asteris Influences Growth of the Nonnatural Host Plant Arabidopsis thaliana
}

\author{
Linda Jahn, ${ }^{1}$ Lisa Storm-Johannsen, ${ }^{1}$ Diana Seidler, ${ }^{1}$ Jasmin Noack, ${ }^{1}$ Wei Gao, ${ }^{2}$ Thomas Schafhauser, ${ }^{1,3}$ \\ Wolfgang Wohlleben, ${ }^{3}$ Willem J. H. van Berkel, ${ }^{4}$ Philippe Jacques, ${ }^{5}$ Tambi Kar, ${ }^{6}$ Birgit Piechulla, ${ }^{7}$ and \\ Jutta Ludwig-Müller ${ }^{1, \dagger}$ \\ ${ }^{1}$ Plant Physiology, Faculty of Biology, Technische Universität Dresden, 01062 Dresden, Germany \\ ${ }^{2}$ Biopsychology, Faculty of Psychology, Technische Universität Dresden, 01062 Dresden, Germany \\ ${ }^{3}$ Interfaculty Institute of Microbiology and Infection Medicine, Microbiology and Biotechnology, Eberhard Karls Universität Tübingen, \\ 72076 Tübingen, Germany \\ ${ }^{4}$ Laboratory of Biochemistry, Wageningen University Dreijenlaan 3, 6703 HA Wageningen, The Netherlands \\ ${ }^{5} \mathrm{MiPI}$, TERRA Teaching and Research Centre, Joint Research Unit BioEcoAgro, UMRt 1158, Gembloux, Belgium \\ ${ }^{6}$ Lipofabrik, Cité Scientifique, Bât. Polytech-Lille, Avenue Langevin 59 655, Villeneuve d'Ascq, France \\ ${ }^{7}$ Institute for Biological Science, Biochemistry, University of Rostock, 18059 Rostock, Germany
}

Accepted 5 October 2021.

\begin{abstract}
Cyanodermella asteris is a fungal endophyte from Aster tataricus, a perennial plant from the northern part of Asia. Here, we demonstrated an interaction of $C$. asteris with Arabidopsis thaliana, Chinese cabbage, rapeseed, tomato, maize, or sunflower resulting in different phenotypes such as shorter main roots, massive lateral root growth, higher leaf and root biomass, and increased anthocyanin levels. In a variety of cocultivation assays, it was shown that these altered phenotypes are caused by fungal $\mathrm{CO}_{2}$, volatile organic compounds, and soluble compounds, notably astins. Astins A, C, and G induced plant growth when they were individually included in the medium. In return, $A$. thaliana stimulates the fungal astin $C$ production during cocultivation. Taken together, our results indicate a bilateral interaction between the fungus and the plant. A stress response in plants is induced by fungal metabolites while plant stress hormones induced astin $\mathrm{C}$ production of the fungus. Interestingly, our results not only show unidirectional influence of the fungus on the plant but also vice versa. The plant is able to influence growth and secondary metabolite production in the endophyte, even when
\end{abstract}

${ }^{\dagger}$ Corresponding author: J. Ludwig-Müller; Jutta.Ludwig-Mueller@tu-dresden.de

Funding: Financial support was provided by the European Union (European Regional Development Fund, ERA-IB Project Astinprod ERA-IB-15-039) and the Free State of Saxony (Sächsische Aufbaubank grants 100271404 and 100271410). L. Jahn, T. Schafhauser, and J. Ludwig-Müller were funded by the Sächsische Aufbaubank. T. Schafhauser was supported by the Institutional Strategy of the University of Tübingen (Project ZUK63). W. Wohlleben was funded by the Bundesministerium für Bildung und Forschung (grant 0315934). W. J. H. van Berkel was supported by the Netherlands Organisation for Scientific Research (Project ACTS 053.80.713).

*The $\boldsymbol{e}$-Xtra logo stands for "electronic extra" and indicates that supplementary figures and supplementary tables are published online.

Conflict of interest: P. Jacques is a co-founder of Lipofabrik and Lipofabrik Belgium and a member of the scientific advisory board of both companies. distributed under the CC BY-NC-ND 4.0 International license. both organisms do not live in close contact, suggesting the involvement of volatile compounds.

Keywords: Arabidopsis thaliana, astins, Cyanodermella asteris, endophyte, fungus-plant interactions, plant-microbe-interaction, secondary metabolism, volatiles

The fungal endophyte Cyanodermella asteris (Stictidaceae) was isolated from the inflorescence axis of the tartaric aster Aster tataricus (Asteraceae) (Jahn et al. 2017). This plant originates from the northern part of Asia (Siberia, Mongolia, north of Japan, and China) and is known for its use in the traditional Chinese medicine because of its antibacterial, antiviral, antiulcer, and diuretic activities (Shao et al. 1997a, b; Shirota et al. 1997). During the 1990s, Morita and colleagues discovered a new substance group in A. tataricus, called astins (Itokawa et al. 1994; Morita et al. 1993a, b, 1994, 1995).

Astins are chlorinated, cyclic pentapeptides of proteinogenic (proline and serine) and nonproteinogenic (allo-threonine, $\alpha$-aminobutyric acid, and $\beta$-phenylalanine) amino acids (Fig. 1). More than 20 different astins are currently known (Morita et al. 1993b; Xu et al. 2013), and nearly all of them are differentially chlorinated at the proline residue $\left(\mathrm{Pro}^{1}\right)$. Only four of them are dichlorinated, which is important for the antitumor activity of astin $\mathrm{A}$ to $\mathrm{C}$ (astin $\mathrm{K}$ is also dichlorinated but the antitumor activity was yet not shown) (Xu et al. 2013).

The fungus $C$. asteris produces astins in culture and, thus, we assumed that the endophyte is the actual organism capable of synthesizing these interesting and valuable metabolites. Interestingly, not all astins mentioned above could be detected in in vitro cultures of C. asteris (Schafhauser et al. 2019). We found only astins $\mathrm{C}, \mathrm{F}$, and $\mathrm{G}$ in $C$. asteris cultures, while the two most prominent other ones, astins $\mathrm{A}$ and $\mathrm{B}$, were only found in the plant $A$. tataricus. This observation suggests that the plant takes up and accumulates the fungal astins, most likely after their secretion by $C$. asteris, and is probably able to modify them. To understand astin production and regulation, we studied the interaction between the plant $A$. tataricus and the fungal endophyte $C$. asteris in more detail. The endophyte lives within the host plant and, thus, it was hypothesized that fungal astin biosynthesis in culture 
can be stimulated and altered by the action of different plant hormones. Phytohormones such as auxins and cytokinins but also stress hormones such as salicylic acid play an important role in the crosstalk between the host plant and the endophyte (Ali et al. 2017). Auxins and cytokinins are shown to be involved in many plant-microorganism interactions of both phytopathogens (Ludwig-Müller et al. 2017; Reineke et al. 2008) as well as plantbeneficial bacteria (Contesto et al. 2010; Dobbelaere et al. 1999; Kochar et al. 2011; Ortíz-Castro et al. 2008) and fungi (ContrerasCornejo et al. 2009; Pons et al. 2020; Salas-Marina et al. 2011).

We searched for a feasible plant-endophyte test system, because $A$. tataricus is impracticable due to its winter dormancy and large size. In addition to different Compositae species such as sunflower, we also tested Chinese cabbage, rapeseed, tomato, maize, and Arabidopsis thaliana. We decided to use A. thaliana for the plant-fungus interaction studies because A. thaliana is a well-studied model plant for plant-pathogen and plant-beneficial microbe studies (Jäschke et al. 2010; Peškan-Berghöfer et al. 2004; Proença et al. 2019). For example, the root-colonizing endophytic fungus Serendipita indica (formerly Piriformospora indica) (Verma et al. 1998) promotes the growth of A. thaliana by improving biomass, chlorophyll content, and lateral root density (Abdelaziz et al. 2017) via modulation of phytohormone levels of auxins and cytokinins (Vadassery et al. 2008; Xu et al. 2018). The plant-growth-promoting bacterium Bacillus megaterium UMCV1 promotes growth of $A$. thaliana by increasing lateral root growth and root hair elongation (López-Bucio et al. 2007) through influencing the cytokinin signaling pathway (Ortíz-Castro et al. 2008). C. asteris produces indole-3-acetic acid, the main auxin in plants, over a tryptophan-dependent and -independent pathway in in vitro cultures and can influence the root phenotype of $A$. thaliana through the additional auxin (Jahn et al. 2021). Despite the influence of fungal auxins, the root phenotype of $A$. thaliana cannot be explained by auxins alone.

In addition to higher molecular weight compounds involved in plant growth promotion, bacteria and fungi also produce small molecular weight volatile organic compounds (VOCs) that stimulate plant growth even over longer distances. Ryu et al. (2003) showed that different Bacillus strains induced plant growth and biomass production in $A$. thaliana, although both organisms were physically separated. In the identical experimental setup with Escherichia coli $\mathrm{DH} 5 \alpha$, these stimulating effects were not

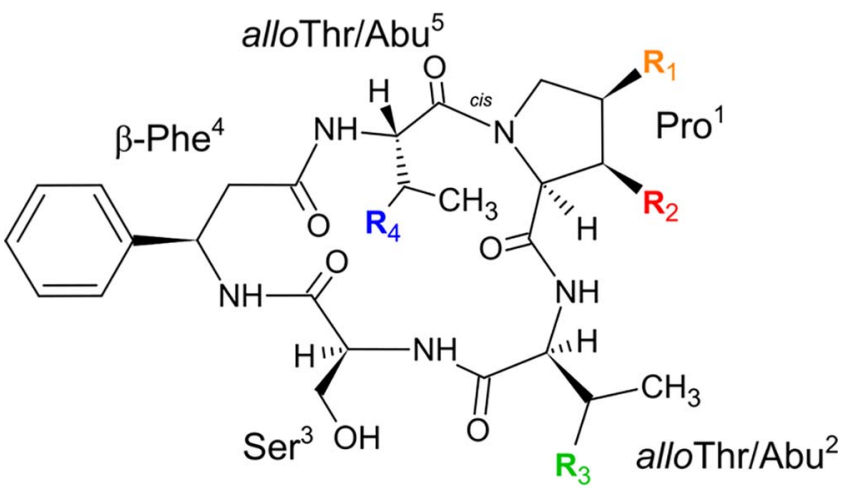

$$
\begin{array}{ll}
\text { astin } A & \mathrm{R}_{1}=\mathrm{Cl}, \mathrm{R}_{2}=\mathrm{Cl}, \mathrm{R}_{3}=\mathrm{H}, \mathrm{R}_{4}=\mathrm{OH} \\
\text { astin } \mathrm{B} & \mathrm{R}_{1}=\mathrm{Cl}, \mathrm{R}_{2}=\mathrm{Cl}, \mathrm{R}_{3}=\mathrm{OH}, \mathrm{R}_{4}=\mathrm{H} \\
\text { astin C } & \mathrm{R}_{1}=\mathrm{Cl}, \mathrm{R}_{2}=\mathrm{Cl}, \mathrm{R}_{3}=\mathrm{H}, \mathrm{R}_{4}=\mathrm{H} \\
\text { astin } \mathrm{G} & \mathrm{R}_{1}=\mathrm{H}, \mathrm{R}_{2}=\mathrm{H}, \mathrm{R}_{3}=\mathrm{H}, \mathrm{R}_{4}=\mathrm{H}
\end{array}
$$

Fig. 1. Basic structure of astins from the plant Aster tataricus and the fungal endophyte Cyanodermella asteris. The cyclic pentapeptides contain proteinogenic (proline and serine) and nonproteinogenic (allo-threonine, $\alpha$-aminobutyric acid, and $\beta$-phenylalanine) amino acids. The Pro residue can be chlorinated. observed. Acetoin was tested and shown to be a key VOC in promoting plant growth (Ryu et al. 2003). In addition to VOCs, inorganic volatile compounds (e.g., carbon dioxide $\left[\mathrm{CO}_{2}\right]$ from fungal respiration) may also influence plant growth. Particularly in closed experimental systems, only little or no gas exchange can take place between the interior of the growth chamber or closed cultivation vessel, such as a Petri dish, and the environmental atmosphere; subsequently emitted organic and inorganic volatile compounds can accumulate to unnaturally high concentrations. It was convincingly shown that $\mathrm{CO}_{2}$ produced by microbes stimulates photosynthesis, growth, and development of the plant during cocultivation (Kai and Piechulla 2009; Van der Kooij et al. 1999), and the effect of $\mathrm{CO}_{2}$ on plants has to be taken into account when cocultivation assays are performed.

Here, we show the promotion of $C$. asteris on the growth and development of $A$. thaliana. We use different cocultivation assays to show the influence of soluble and volatile fungal compounds on A. thaliana. Not only did the fungal partner C. asteris affect the plant partner but also $A$. thaliana influenced the fungal growth and metabolism. Therefore, our interaction studies with A. thaliana suggest a contribution of soluble astins, VOCs, and $\mathrm{CO}_{2}$ from $C$. asteris.

\section{RESULTS}

\section{Selection of the plant species}

for fungus-plant cocultivations.

The natural host plant of $C$. asteris is Aster tataricus. However, its availability and growth in the laboratory or greenhouse has several limitations. To find a feasible host, sunflower, Chinese cabbage, rapeseed, tomato, and Arabidopsis thaliana were cocultivated with $C$. asteris. All plant species showed the same altered growth phenotype in these experiments when they were inoculated with $C$. asteris (Supplementary Fig. S2). Because most plant species grew too large for sterile in vitro cocultivation, the following experiments were performed with A. thaliana.

\section{Development of a medium for cocultivation of A. thaliana and C. asteris.}

To find alternatives for studying the interaction of the tartaric aster endophyte $C$. asteris and a plant host, we have established and optimized cocultivation conditions. The endophyte and the plant do not grow well on the same culture medium. Smaller plants such as $A$. thaliana grow well in Petri dishes with various tissue culture media, preferably Murashige and Skoog (MS) medium (Murashige and Skoog 1962), while C. asteris grows well on malt extract (MEA) medium (Schafhauser et al. 2019). Different media combinations were tested. A. thaliana developed reduced rosette diameter, shorter main roots, and less leaf material in Petri dishes without any MS medium compared with growth on medium containing MS salts and vitamins (Supplementary Table S1). The fungus was able to grow well on half-strength MS medium when it was supplemented with the components that constitute the previously established (Schafhauser et al. 2019) MEA medium (MEAlow). Therefore, a cocultivation medium composed of half-strength MS without sucrose plus MEAlow (1/2 MS/MEAlow) was used for the experiments described in this research.

\section{Cocultivation of $C$. asteris and $A$. thaliana in a closed system.}

A. thaliana was cocultivated with $C$. asteris in nonseparated Petri dishes (diameter of $9 \mathrm{~cm}$ ) sealed with Parafilm. Different distances $(1.5,3.0$, and $4.5 \mathrm{~cm})$ between the conidia (inoculation point) and the hypocotyl of the plants were tested. After 35 days, A. thaliana developed an altered root phenotype when cocultivated with $C$. asteris that consisted of shorter roots but 
produced a massive number of lateral roots (Fig. 2A and C). Furthermore, rosettes as well as roots had a larger biomass (Fig. 2B and D) and flowering occurred earlier than in the plants of noninoculated controls (Fig. 2E). The phenotype was also very similar between $A$. thaliana and $C$. asteris plants when inoculated at distance of 4.5 and $7.5 \mathrm{~cm}$ in squared Petri dishes $(12$ by $12 \mathrm{~cm}$ ) (Supplementary Fig. S3). With a larger distance between plants and fungi, the main root length development was more similar to the control (Fig. 2A; Supplementary Fig. S3A). C. asteris increased the biomass production of $A$. thaliana at a $7.5-\mathrm{cm}$ distance compared with the noninoculated controls (Supplementary Fig. S3B).

The increase in root biomass was mainly due to massive production of lateral roots (Fig. 2C and D). The plants with the closest distance to $C$. asteris had the lowest biomass in all treatments $(1.5 \mathrm{~cm})$ (Supplementary Fig. S3). Rosettes and roots with a larger distance of 3.0 and $4.5 \mathrm{~cm}$ had similar biomasses of rosettes and roots. Effects on the main root length were the same as those observed for root biomass. The further away the fungus was, the better the main root grew but the fewer lateral roots were formed (Fig. 2C). Once the roots and the fungus were in direct contact, the roots did not grow over the mycelium of $C$. asteris (Fig. 2C). The plants treated with $C$. asteris developed faster than the controls (Fig. 2E). All plants with a distance of $4.5 \mathrm{~cm}$ flowered 35 days after sowing, while the other plants growing closer to $C$. asteris flowered faster than the control but not as fast as the ones growing at a distance of $4.5 \mathrm{~cm}$ to the fungal mycelium (Supplementary Fig. S3C).

The altered phenotypes of $A$. thaliana in the cocultivations are proposed to be due to volatiles or soluble substances of C. asteris. In the following sections, three possible fungal compound classes $\left(\mathrm{CO}_{2}\right.$, VOCs, and astins) that could be responsible for generating the abovedescribed phenotypes will be addressed.

\section{Fungal $\mathrm{CO}_{2}$ promotes plant growth of $A$. thaliana.}

Plants were cocultivated with $C$. asteris in a closed system (sealed with Parafilm) to show the effect of low molecular weight volatile substances. These cocultivation experiments were performed in tripartite Petri dishes (Fig. 3; Supplementary Figs. S5 and S6). One of these low molecular weight substances was presumed to be the inorganic volatile $\mathrm{CO}_{2}$ released from the fungus. The volatile $\mathrm{CO}_{2}$ was trapped by barium hydroxide $\left[\mathrm{Ba}(\mathrm{OH})_{2}\right]$ to evaluate the growth effect.
A

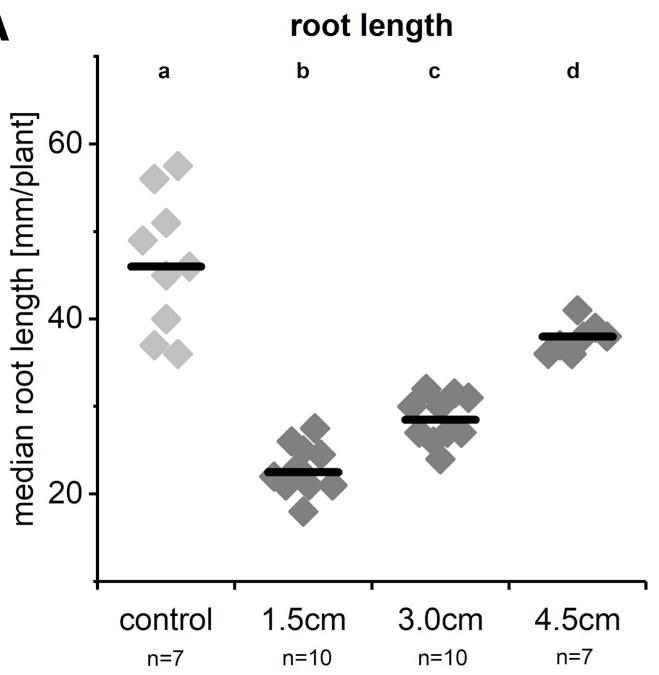

D

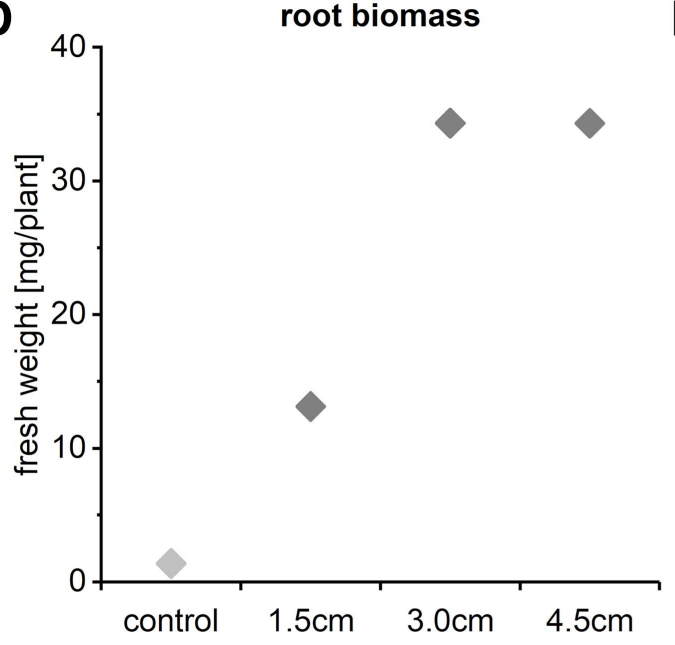

B

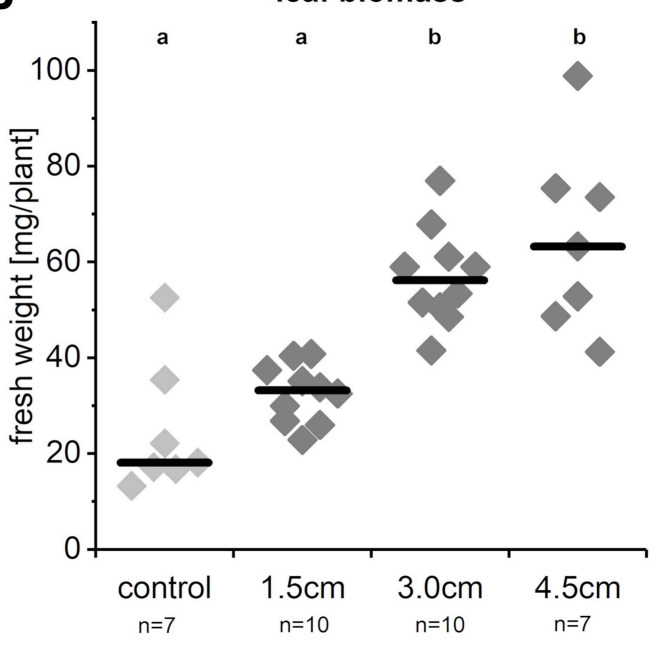

E

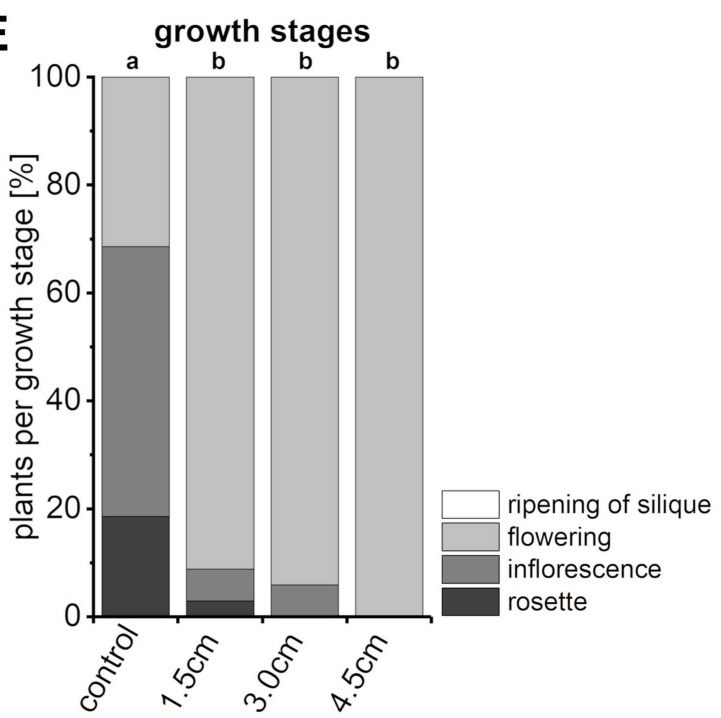

C control

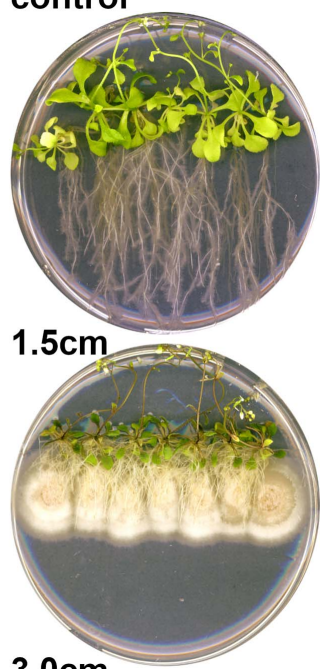

$3.0 \mathrm{~cm}$

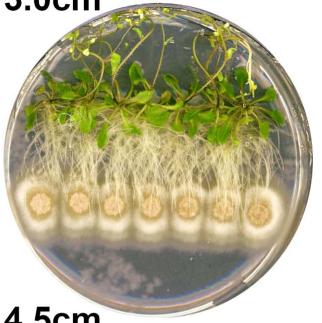

$4.5 \mathrm{~cm}$

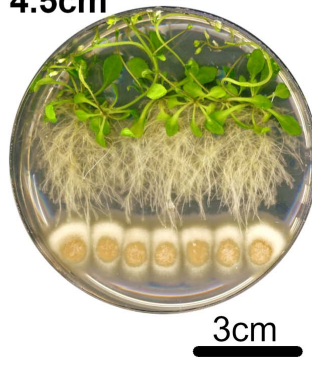

Fig. 2. Cocultivation of Arabidopsis thaliana with Cyanodermella asteris in in 9-cm plates without partitions and sealed with Parafilm. Cocultivation was on half-strength Murashige and Skoog plus malt extract without sucrose (1/2 MS/MEAlow) medium under long-day conditions for 35 days. $C$. asteris was inoculated at a distance of $1.5,3.0$, and $4.5 \mathrm{~cm}$ from $A$. thaliana. Growth of $A$. thaliana is shown. $\mathbf{A}, \mathbf{M a i n}$ root length $\left(P<0.001, \eta_{\mathrm{p}}{ }^{2}=0.924\right)$; $\mathbf{B}$, leaf biomass $\left(P<0.001, \eta_{\mathrm{p}}{ }^{2}=0.805\right)$; and $\mathbf{C}$, pictures of cocultivation of $A$. thaliana and $C$. asteris. D, Root biomass of noninoculated and inoculated plants. E, Plant development of $A$. thaliana cocultivated with $C$. asteris $\left(P<0.001, \mathrm{n}^{2}=0.448\right)$. Significant differences are labeled with lower case letters $(\mathrm{a}, \mathrm{b}$, c) and bar indicates median. 
Plants growing in tripartite Petri dishes with $C$. asteris produced more leaf and root biomass than noninoculated plants, regardless of whether or not $\mathrm{Ba}(\mathrm{OH})_{2}$ was present (Fig. 3B; Supplementary Fig. S5). The addition of $\mathrm{Ba}(\mathrm{OH})_{2}$ decreased the root biomass in control and $C$. asteris-treated plants compared with plants without $\mathrm{Ba}(\mathrm{OH})_{2}$. In the control experiments, where plants were incubated with $\mathrm{Ba}(\mathrm{OH})_{2}$ and without cocultivation with the fungus, leaf and root biomass decreased massively. The plants developed very poorly (e.g., no new leaf development was observed after 14 days of cultivation, inflorescence was decelerated, flowering and ripening of siliques occurred, and plants died after 28 days of cultivation) (Fig. 3A; Supplementary Fig. S6). Due to the weak growth, plant material of all Petri dishes was pooled for weighting. In cocultivation experiments of plants with C. asteris in the presence of $\mathrm{Ba}(\mathrm{OH})_{2}$, C. asteris could compensate the $\mathrm{CO}_{2}$ complexation of $\mathrm{Ba}(\mathrm{OH})_{2}$. This was indicated by a decreased root biomass of $A$. thaliana cocultivated with $C$. asteris and $\mathrm{Ba}(\mathrm{OH})_{2}$, which was, however, still higher than in plants without any treatment (Fig. 3B).

If the fungal inoculum was less dense (only two spots, each with $10^{5}$ or $10^{3}$ spores), then $A$. thaliana developed a phenotype similar to the control without $C$. asteris under the $\mathrm{Ba}(\mathrm{OH})_{2}$ condition (data not shown).

In summary, these experiments clearly indicate that fungal $\mathrm{CO}_{2}$ of $C$. asteris promoted plant growth of $A$. thaliana, which was documented by the increased leaf and root biomass as well as various parameters of plant development (Fig. 3; Supplementary Figs. S5 and S6). To eliminate or minimize the $\mathrm{CO}_{2}$ effect in the following fungus-plant interaction studies, we used Petri dishes which were closed by a MaiMed tape that allows diffusion of small molecules (MaiMed 2011). It was hypothesized that, in these experiments, low molecular weight organic and inorganic volatiles could diffuse between the Petri dishes and the environment. The principal idea was tested with bipartite Petri dishes containing $\mathrm{Ba}(\mathrm{OH})_{2}$ in one compartment and medium plus plant or fungus in the other compartment. The Petri dishes were sealed either with Parafilm, MaiMed tape, or nothing and the amount of barium carbonate $\left(\mathrm{BaCO}_{3}\right)$ was determined after 28 days, representing the amount of $\mathrm{CO}_{2}$ complexed by $\mathrm{Ba}(\mathrm{OH})_{2}$ (Fig. 4). The $\mathrm{BaCO}_{3}$ levels were higher in setups where the fungus was growing, indicating that $\mathrm{CO}_{2}$ was generated by respiration of the fungus. The $\mathrm{BaCO}_{3}$ level in Petri dishes with $A$. thaliana or without organisms reached approximately the same amount regardless of whether the Petri dishes were unsealed or sealed with MaiMed tape or Parafilm. In Petri dishes with A. thaliana or without an organism, sealing with Parafilm led to a decreased $\mathrm{BaCO}_{3}$ level.

The results supported the idea that the MaiMed tape can be used to mimic an open system. Therefore, Petri dishes were taped with MaiMed tape in all further experiments to keep the Petri dishes sterile and the medium moist over a long incubation time. In the cocultivation experiment, $A$. thaliana plants inoculated with $C$. asteris showed less root growth but an increased root biomass (Fig. 5; Supplementary Figs. S4 and S5). However, C. asteris no longer influenced the plant reproductive development, seen also in a similar leaf biomass (Supplementary Fig. S6).

Inoculation of $A$. thaliana with dead (= autoclaved) spores or hyphae had no effect on the plants. The inoculated plants

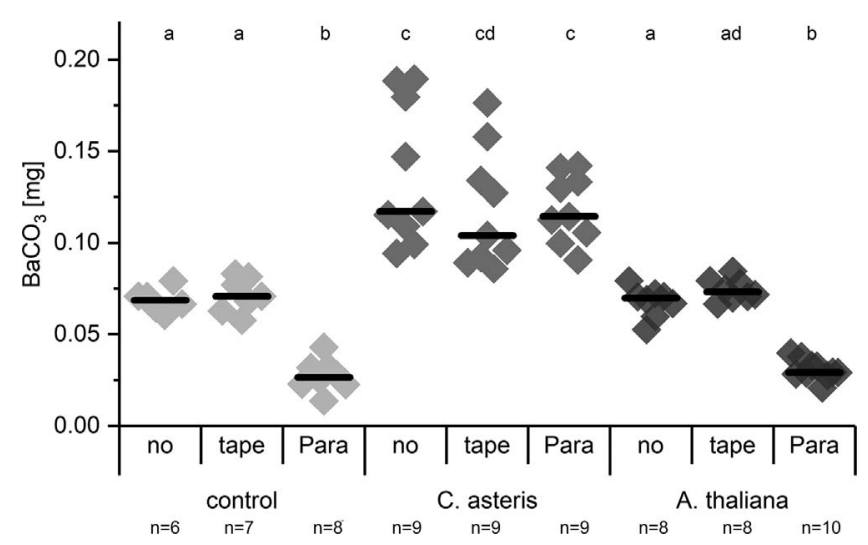

Fig. 4. Accumulation of $\mathrm{CO}_{2}$ in 9-cm Petri dishes with or without the presence of Cyanodermella asteris or Arabidopsis thaliana. The Petri dishes without partitions, containing half-strength Murashige and Skoog plus malt extract without sucrose (1/2 MS/MEAlow) medium inoculated with C. asteris, A. thaliana, or without any organisms were closed without tape (no), with MaiMed (tape), or with Parafilm (Para). The $\mathrm{BaCO}_{3}$ amount of each Petri dish was determined after 28 days $(n=6$ to 10 , $\left.P<0.001, \mathrm{n}_{\mathrm{p}}{ }^{2}=0.952\right)$. Significant differences are labeled with lower case letters $(\mathrm{a}, \mathrm{b}, \mathrm{c})$ and bar indicates median.
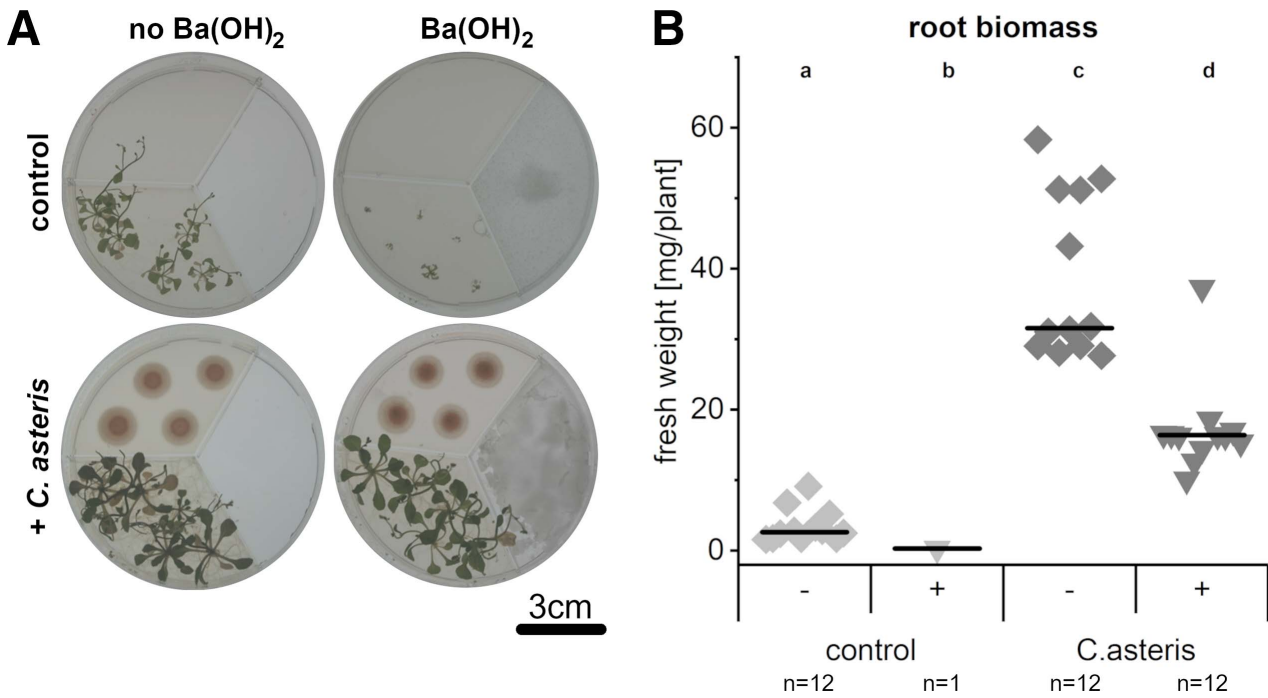

Fig. 3. Cocultivation of Arabidopsis thaliana with Cyanodermella asteris in 9-cm tripartite plates in the presence or absence of $\mathrm{Ba}(\mathrm{OH})_{2}$ and sealed with Parafilm. Cocultivation was on half-strength Murashige and Skoog plus malt extract without sucrose (1/2 MS/MEAlow) medium under long-day conditions for 35 days. A, Growth of A. thaliana and C. asteris in the presence of $\mathrm{Ba}(\mathrm{OH})_{2}$. B, Root biomass of A. thaliana plants noncocultivated (control) or cocultivated with $C$. asteris in the absence $(-)$ or presence $(+)$ of $\mathrm{Ba}(\mathrm{OH})_{2}\left(P<0.001, \mathrm{\eta}_{\mathrm{p}}{ }^{2}=0.921\right)$. Significant differences are labeled with lower case letters (a, b, c, d) and bar indicates median. 
developed like the controls without $C$. asteris, indicating that the living fungus is necessary for the growth phenotype reported here (data not shown).

\section{VOCs affect plant growth of $A$. thaliana.}

As reported above, part of the growth-promoting effect in separated cocultivation is due to $\mathrm{CO}_{2}$ released by the fungus; however, the growth effects observed cannot only be assigned to a $\mathrm{CO}_{2}$ effect. Because VOCs as well as highly molecular-soluble compounds of $C$. asteris might also affect the growth of $A$. thaliana, we examined both hypotheses in cocultivation experiments using bipartite Petri dishes sealed with MaiMed tape.

Growth of $A$. thaliana was enhanced under these conditions (Fig. 6). Plants cocultivated with $C$. asteris produced more roots (Fig. 6A and B) and leaves (Supplementary Fig. S5). The root length (Supplementary Fig. S4) and plant development (Supplementary Fig. S6) of A. thaliana were not significantly influenced by the presence of $C$. asteris, as was observed in nonseparated Petri dishes (Fig. 5). The increased biomass of leaves (1.67-fold) and roots (2.88-fold) indicated a direct or indirect (via agar) influence of as-yet-unidentified fungal VOCs on plant growth promotion.

\section{C. asteris induces a stress response in $A$. thaliana.}

Because $C$. asteris is not a natural endophyte of $A$. thaliana, anthocyanin and chlorophyll levels of plants were determined as general stress indicators. However, such a defense response could also be found in the interaction of a natural endophyte and its host plant (Mandyam et al. 2013; Schulz and Boyle 2005). Cocultivation of $A$. thaliana with the nonnatural endophyte $C$. asteris led to an increased anthocyanin level in plants (Table 1; Supplementary Fig. S7). The anthocyanin content was highly increased (Parafilm: 9.7- to 57.6-fold; MaiMed tape: 3.0-fold) in plants that had direct contact with C. asteris, regardless of which sealing was used. Plants that were inoculated $3.0 \mathrm{~cm}$ away from the hypocotyl had a higher anthocyanin
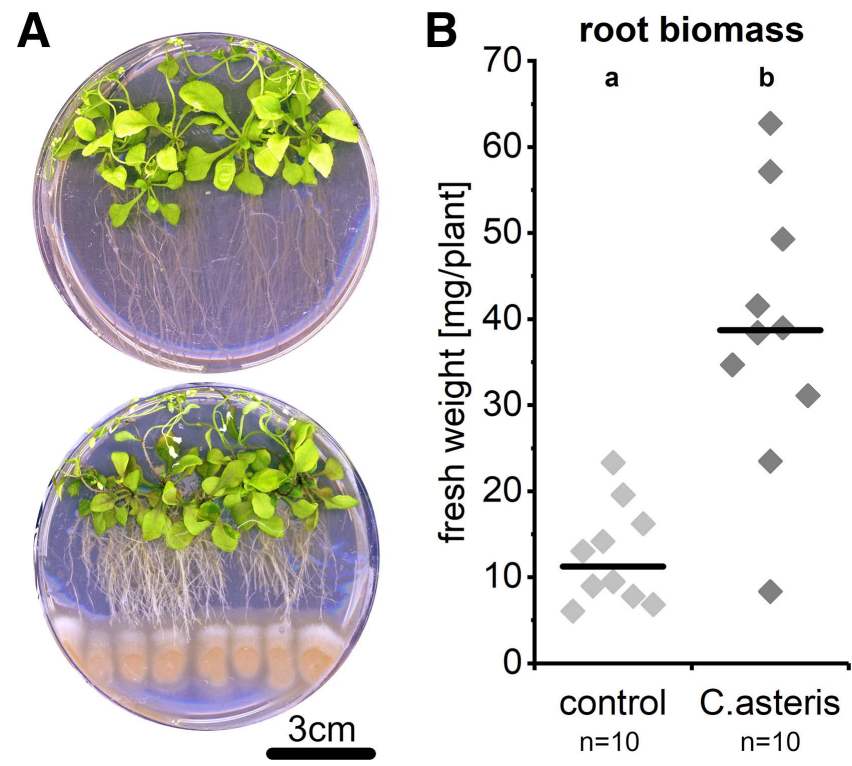

Fig. 5. Cocultivation of Arabidopsis thaliana with Cyanodermella asteris in 9-cm plates without partitions, sealed with MaiMed. Cocultivation was on half-strength Murashige and Skoog plus malt extract without sucrose (1/2 MS/MEAlow) medium under long-day conditions for 35 days. C. asteris was inoculated at a distance of $4.5 \mathrm{~cm}$ from A. thaliana seedlings. A, Growth of A. thaliana without and with $C$. asteris on nonseparated plates. B, Root biomass of $A$. thaliana without (control) and with $C$. asteris $\left(P<0.001, \mathrm{n}^{2}=0.543\right)$. Significant differences are labeled lower case letters $(\mathrm{a}, \mathrm{b})$ and bar indicates median. content than plants with a distance of $1.5 \mathrm{~cm}$. Because these plants were quickly overgrown by $C$. asteris, they were much smaller and could not develop as much anthocyanins as larger plants. The addition of $\mathrm{Ba}(\mathrm{OH})_{2}$ did not change the anthocyanin level in A. thaliana. Only plants cocultivated with $C$. asteris showed a higher anthocyanin level, which reduced to the control level in the presence of $\mathrm{Ba}(\mathrm{OH})_{2}$. A separation of plant and fungus on bipartite Petri dishes led to a slight decrease in the anthocyanin level, as long as the Petri dishes were tightly closed with Parafilm. Cocultivated plants without any influence of fungal soluble or volatile compounds (bipartite and MaiMed tape) had anthocyanin levels similar to those of the respective controls without any fungus.

We also measured the chlorophyll content in A. thaliana plants cocultivated with $C$. asteris on different Petri dishes (Table 1; Supplementary Fig. S8). C. asteris had no strong effect on the chlorophyll content of cocultivated plants under almost all conditions, except when plants were cocultivated with $C$. asteris on bipartite Petri dishes sealed with Parafilm. In this case, a massive decrease in chlorophyll content was observed. If the fungus was in direct contact with $A$. thaliana and in plates sealed with MaiMed tape, the chlorophyll content of the leaves was slightly decreased.

A. thaliana mutants defective in the biotic stress pathways were cocultivated with $C$. asteris in the open system to investigate the possible involvement of stress-associated plant hormones salicylic acid (SA), jasmonic acid (JA), and ethylene that could lead to the phenotype caused by $C$. asteris. NPR1 is a key regulator in the systemic acquired resistance in plants induced by SA (Cao et al. 1997), SID2 is an enzyme in SA biosynthesis (Wildermuth et al. 2001) and NahG encodes a bacterial SA hydroxylase, degrading SA (Friedrich et al. 1995). Consequently, all mutants are, to some extent, impaired in their response to SA. The nprl-1 and sid2-1 mutants and NahG overexpressing plants of $A$. thaliana developed similarly to the control in the presence of $C$. asteris (Supplementary Fig. S9), indicating that $C$. asteris is not using the SA pathway to induce
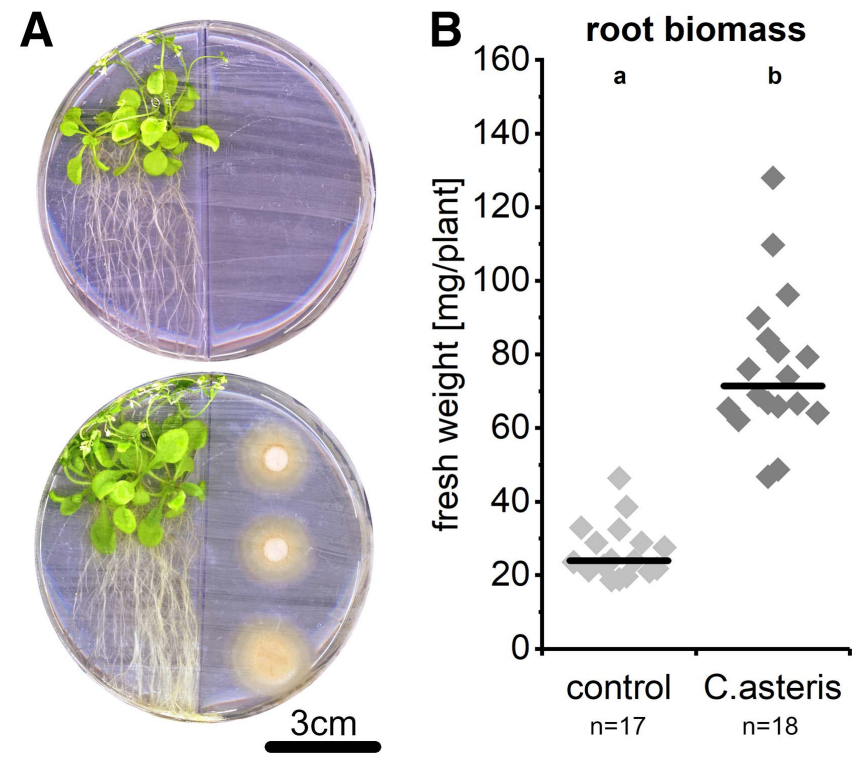

Fig. 6. Cocultivation of Arabidopsis thaliana with Cyanodermella asteris in 9-cm bipartite plates, sealed with MaiMed. Cocultivation was on halfstrength Murashige and Skoog plus malt extract without sucrose (1/2 MS/MEAlow) medium under long-day conditions for 35 days. A, Growth of $A$. thaliana without and with $C$. asteris in cocultivation. B, Root biomass produced by $A$. thaliana without (control) and with $C$. asteris $(C$. asteris) in cocultivation $\left(P<0.001, \mathrm{n}^{2}=0.723\right)$. Significant differences are labeled with lower case letters $(a, b)$ and bar indicates median. 
the altered phenotype of $A$. thaliana. The jarl-1 mutant, which is defective in the JA response (Staswick et al. 1992), and the etrl-1 mutant, which cannot perceive the ethylene signal (Gamble et al. 2002), showed also no alterations in the phenotype of $A$. thaliana inoculated with $C$. asteris compared with wild-type plants (Supplementary Fig. S9).

\section{Astins induce growth of $A$. thaliana.}

Astins are secondary metabolites from $C$. asteris, known also from its host plant Aster tataricus. A biological effect on plants has not been reported yet. To investigate possible biological effects of astins on Arabidopsis thaliana, the plants were cultivated on MS medium containing different amounts (10 pM to $10 \mu \mathrm{M}$ ) of astins A, C (both dichlorinated), or G (nonchlorinated) (Fig. 1). As discussed in the introduction, astins $\mathrm{A}$ and $\mathrm{C}$ differ in a hydroxyl group at the fifth amino acid (allo $\left.\mathrm{Thr}^{5} \rightarrow \mathrm{Abu}^{5}\right)$. Astin A can only be found in Aster tataricus together with $C$. asteris and not in fungal cultures alone (Schafhauser et al. 2019). Our experiments showed that all three tested astins influenced the growth and development of Arabidopsis thaliana during 35 days of cultivation (Fig. 7; Supplementary Figs. S10 and S11).

The plant growth (root length and rosette diameter) of $A$. thaliana was significantly inhibited at $10 \mu \mathrm{M}$ astin A over the whole period. Lower concentrations of astin A did not suppress root growth (Fig. 7; Supplementary Fig. S10) or rosette growth (Fig. 7; Supplementary Fig. S11) and even induced plant growth below an astin A concentration of $10 \mu \mathrm{M}$ (Fig. 7). The growth induction of A. thaliana by astin A concentrations less than $10 \mu \mathrm{M}$ was accompanied by faster plant development (e.g., earlier flowering) (Supplementary Fig. S12). A. thaliana plants grown with different concentrations of astin $\mathrm{C}$, as determined after 7 days, suppressed root growth at all concentrations (Supplementary Fig. S10). This inhibition turned into a slight growth promotion after 35 days of cultivation (Supplementary Fig. S10). Astin C increased the rosette diameter at all astin $\mathrm{C}$ concentrations throughout the 35 days of cultivation (Fig. 7; Supplementary Fig. S11). Astin C plant growth enhancement was similar to that of astin A, and which was accompanied by faster reproductive plant development (Supplementary Fig. S12). The nonchlorinated astin G affected root length and rosette diameter, and flowering started slightly earlier at concentrations higher than $1 \mathrm{nM}$.

In summary, astins, especially the dichlorinated astins A and $\mathrm{C}$, led to a faster development and, therefore, an earlier flowering of $A$. thaliana compared with the treatment with astin $\mathrm{G}$ and with untreated plants. Because $C$. asteris produces only astins C, F, and G (Schafhauser et al. 2019), it is hypothesized that astin $\mathrm{C}$ functions as a bioactive soluble compound that influenced the development of $A$. thaliana in in vitro experiments.

\section{A. thaliana induces astin $\mathrm{C}$ production of $C$. asteris.}

The astin $\mathrm{C}$ production of $C$. asteris was investigated in Petri dishes where the fungus had direct contact with the nonnatural host plant $A$. thaliana (Fig. 8). The astin $\mathrm{C}$ level of $C$. asteris without plants (control) differed in Petri dishes with different sealings: Parafilm led to a higher astin $\mathrm{C}$ production in $C$. asteris than the MaiMed tape. The closer the fungus and A. thaliana were on

A
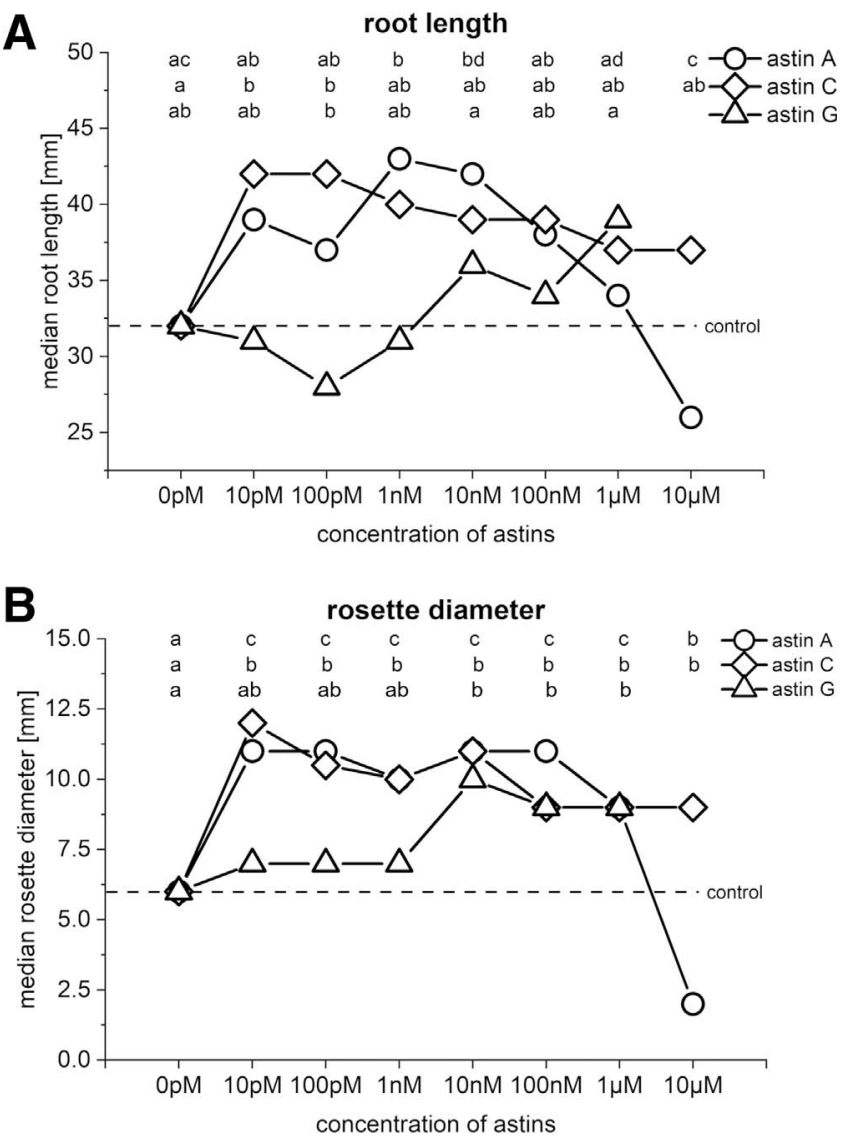

Fig. 7. Growth of main root and rosette of Arabidopsis thaliana with different concentrations of astins A, C, or G. Plants of A. thaliana grew in 9-cm plates without partitions on half-strength Murashige and Skoog plus malt extract without sucrose (1/2 MS/MEAlow) medium including different astins under long-day conditions for 35 days. A, Growth of main root $(P=0.01)$ and $\mathbf{B}$, rosette growth after 35 days of cultivation with different astins $(P=0.01)$. Control plants grown without any astins are shown in sample $0 \mathrm{pM}$. Significant differences are labeled with lower case letters $(\mathrm{a}, \mathrm{b}, \mathrm{c})$.

Table 1. Influence of Cyanodermella asteris on the anthocyanin and chlorophyll content in Arabidopsis thaliana in cocultivation ${ }^{\text {a }}$

\begin{tabular}{|c|c|c|c|c|c|c|}
\hline \multirow[b]{2}{*}{ Experimental settings } & & & \multicolumn{2}{|c|}{ Anthocyanins } & \multicolumn{2}{|c|}{ Chlorophyll } \\
\hline & & & $P$ value & Fold & $P$ value & Fold \\
\hline \multirow[t]{4}{*}{ Nonseparated } & Parafilm & $1.5 \mathrm{~cm}$ & $<0.001$ & $34.5 x$ & 0.246 & - \\
\hline & & $3.0 \mathrm{~cm}$ & - & $57.6 x$ & - & - \\
\hline & & $4.5 \mathrm{~cm}$ & - & $9.7 x$ & - & - \\
\hline & MM tape & $4.5 \mathrm{~cm}$ & 0.005 & $3.97 x$ & 0.029 & $0.70 \times$ \\
\hline \multirow[t]{2}{*}{ Bipartite } & Parafilm & $4.5 \mathrm{~cm}$ & 0.030 & $0.13 x$ & 0.007 & $0.12 \times$ \\
\hline & MM tape & $4.5 \mathrm{~cm}$ & 0.770 & $1.02 x$ & 0.770 & $0.94 \times$ \\
\hline \multirow{3}{*}{ Tripartite with $\mathrm{Ba}(\mathrm{OH})_{2}$} & Control & $\mathrm{Ba}(\mathrm{OH})_{2}$ & 0.162 & $\mathrm{NC}$ & 0.298 & $\mathrm{NC}$ \\
\hline & + C. asteris & $\mathrm{No} \mathrm{Ba}(\mathrm{OH})_{2}$ & - & $30.47 x$ & - & $1.30 x$ \\
\hline & + C. asteris & $\mathrm{Ba}(\mathrm{OH})_{2}$ & - & $5.71 \times$ & - & $1.26 x$ \\
\hline
\end{tabular}


the Petri dishes $(1.5$ and $3 \mathrm{~cm})$, the more astin $C$ was secreted by the fungus. At a distance of $4.5 \mathrm{~cm}$ between $A$. thaliana and the fungus, the fungus produced levels of astin $\mathrm{C}$ similar to that in the control. Astin A, only isolated from the host plant of $C$. asteris (Aster tataricus) (Schafhauser et al. 2019), was not detected under any of these conditions.

\section{Plant hormones induce fungal growth and astin $\mathrm{C}$ production of $C$. asteris.}

Because the original habitat of $C$. asteris is within a host plant, we investigated whether plant signals can influence growth and astin production of $C$. asteris in in vitro cultures. $C$. asteris was cultivated with different plant hormones. The growth of $C$. asteris, based on dry weight determination and astin $\mathrm{C}$ production, was altered by two auxins-1-naphthaleneacetic acid (NAA) and indole-3-acetic acid (IAA) (Fig. 9) — while the two stress hormones SA and JA increased astin $\mathrm{C}$ production but did not alter the fungal growth (Fig. 10).

The synthetic auxin NAA led to an optimum growth curve, with the maximum at $100 \mathrm{nM}$ NAA. The growth was only inhibited at the two highest NAA concentrations of $100 \mu \mathrm{M}$ and $1 \mathrm{mM}$. The natural auxin IAA did not enhance growth of $C$. asteris but reduced growth at the two highest concentrations (inhibition at $100 \mu \mathrm{M}$ and $1 \mathrm{mM}$ ). The two stress hormones SA and JA did not influence fungal growth, except for a growth inhibition at high SA concentrations of $100 \mu \mathrm{M}$ and $1 \mathrm{mM}$. Most notable was the increased astin $\mathrm{C}$ production at a concentration of $10 \mu \mathrm{M} \mathrm{JA}$.

\section{DISCUSSION}

The recently isolated novel endophyte $C$. asteris (Jahn et al. 2017) was cocultivated with Arabidopsis thaliana to investigate the influence of the fungus on the growth and development of this alternative host plant. A. thaliana developed strong phenotypic effects in the presence of $C$. asteris: shorter main roots, massive lateral root growth leading to higher root biomass, and increased leaf biomass (Fig. 2). The term "nonnatural host" we have chosen reflects that an interaction between the two organisms occur: (i) the living fungus is necessary for the interaction, (ii) there is most likely not a classical defense reaction involved, and (iii) the partner plant A. thaliana is influenced by fungal compounds in growth and development. Dead spores and hyphae of $C$. asteris had no effect on A. thaliana, indicating that the bioactive compounds responsible for the plant phenotype have to be produced by the fungus. Growth promotion in nonnatural host plants has already been shown for many other fungi such as S. indica (Singhal et al. 2017; Xu et al. 2018), Trichoderma atrovirida, T. virens (Contreras-Cornejo et al. 2009; González-Pérez et al. 2018), Aspergillus ustus (SalasMarina et al. 2011), and Oidiodendron maius (Casarrubia et al. 2016).

To determine the relevant bioactive compound or compounds in addition to fungal IAA of $C$. asteris that influenced root growth positively (Jahn et al. 2021), inorganic and organic volatiles as well as soluble astins were investigated in cocultivation experiments. Our results of cocultivation studies of $A$. thaliana and $C$. asteris indicated that plant growth promotion was largely caused by $\mathrm{CO}_{2}$, but not exclusively (Figs. 3 and 5). Similar observations were already made in other experimental setups, especially in closed systems (Kai and Piechulla 2009). When Petri dishes are sealed (e.g., with Parafilm), fungal organic and inorganic volatiles will be trapped inside the Petri dish. $\mathrm{CO}_{2}$ that is produced in the cellular respiration can directly be used by the plant in photosynthesis, leading to a higher biomass and stronger plants (Kai and Piechulla 2009; Makino and Mae 1999; Piechulla and Schnitzler 2016). An elevated atmospheric $\mathrm{CO}_{2}$ level increases shoots as well as roots and altered root architecture in agricultural crops (Madhu and Hatfield 2013). The degree to which extent fungal $\mathrm{CO}_{2}$ might affect host growth in planta has still to be answered and remains unsolved.

Detailed cocultivation experiments of A. thaliana plants with $C$. asteris also showed a higher biomass production in Petri dishes sealed with MaiMed tape, suggesting that, additionally, VOCs may promote growth (Fig. 6). $\beta$-Caryophyllene (Minerdi et al. 2011), 3-methyl-1-butanol, 1-decene, and 2-heptylfuran from Trichoderma spp. (Lee et al. 2019) are a few examples of VOCs increasing plant growth (Piechulla et al. 2017). In addition to plant growth promotion, VOCs (e.g., isobutyric acid, benzyl aldehyde, or 6-pentyl- $\alpha$-pyrone) also show antimicrobial activities (Morath et al. 2012) or enhance and prime the plant defense system (Ryu et al. 2003), action modes which are beneficial for plants. A. thaliana, for example, showed an increased level of defense genes in the presence of fungal VOCs such as 1-octen-3-ol, allo-ocimene, or C6-aldehydes and, thus, can react much faster to pathogen attacks (Kishimoto et al. 2006a, b, 2007). A mixture of VOCs of Muscodor albus, an endophytic fungus in the bark of Cinnamomum zeylanicum (cinnamon tree) (Worapong et al. 2001), inhibited or killed phytopathogenic bacteria and fungi (Strobel et al. 2001). Additionally, M. albus can
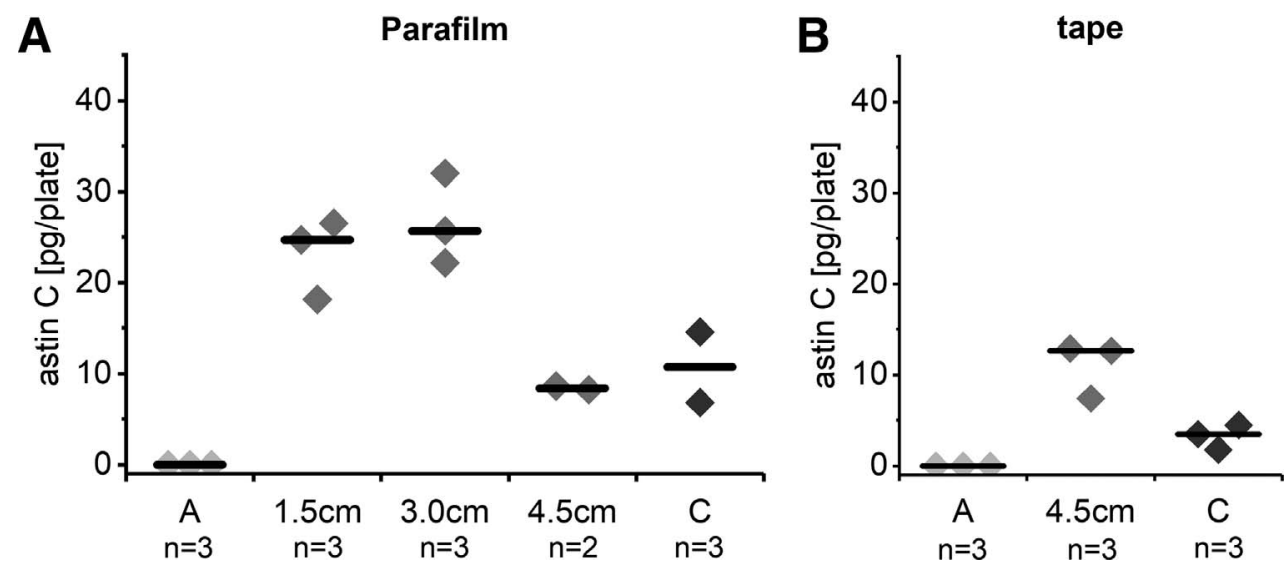

Fig. 8. Astin C production by Cyanodermella asteris in cocultivation with Arabidopsis thaliana on 9-cm plates without partitions. Cocultivation was on half-strength Murashige and Skoog plus malt extract without sucrose (1/2 MS/MEAlow) medium under long-day conditions for 35 days. Plates were sealed with A, Parafilm or $\mathbf{B}$, MaiMed. Astin C concentration was measured in the medium of controls $(\mathrm{A}=$ only A. thaliana and $\mathrm{C}=$ only $C$. asteris $)$ and of cocultivation plates where both organisms were placed at different distances $(1.5,3.0$, and $4.5 \mathrm{~cm})$. Three plates were pooled for each astin $\mathrm{C}$ extraction; bar indicates median. 
control phytopathogens such as Rhizoctonia solani and Phytophthora capsici in soils (Mercier and Manker 2005). It is likely that the plant-associated fungus $C$. asteris is able to influence growth and defense responses in Aster tataricus as well as in the nonnatural host plant Arabidopsis thaliana. Because the MaiMed tape used in these experiments is only an approach to mimic an open system (because the plates had to be cultivated vertically to analyze the root growth), it has to be taken into account that the $\mathrm{CO}_{2}$ content could be increased in these systems. Measuring the $\mathrm{CO}_{2}$ level directly in the cocultivation system would show if there is still a slightly higher $\mathrm{CO}_{2}$ content in the Petri dishes, which still would contribute to the increased biomass production in A. thaliana. The VOCs that additionally cause changes in plant growth need to be identified to investigate their effect during the cocultivation of both partners. Furthermore, it is of interest to learn whether the plant partner might also influence the fungus by producing plant VOCs. Identifying the VOCs responsible for the altered growth phenotype of $A$. thaliana after inoculation with $C$. asteris will be subject of future research.

In addition to small volatile compounds, higher molecular soluble compounds, typically secondary metabolites produced by the endophyte, have to be considered to interact with plant metabolism. Fungi secrete various mixtures of different soluble

A
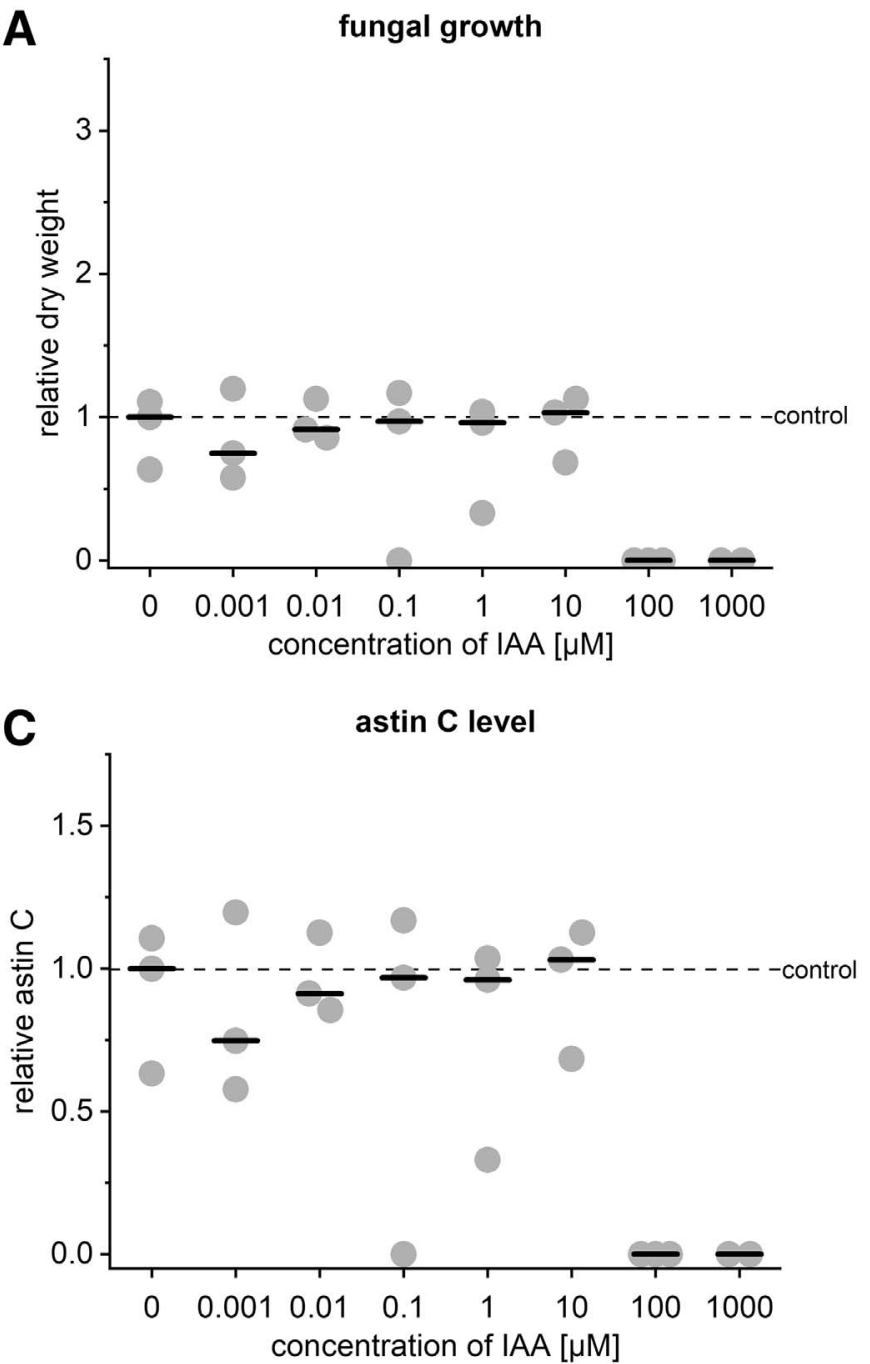

compounds and some of them suppress the growth of the (nonnatural host) plant (Dovana et al. 2015). One group of such secondary metabolites are fungal phytotoxins that are secreted into the medium and inhibit plant growth or induce a stress response in plants. The endophytic fungus Edenia gomezpompae from Callicarpa acuminata leaves (Gonzalez et al. 2007), for example, inhibited growth of Solanum lycopersicum, Echinochloa crus-galli, and Amaranthus hypochondriacus by different phytotoxins (e.g., palmarumycin $\mathrm{EG}_{1}$ and preussomerin $\mathrm{EG}_{4}$ ) (Macías-Rubalcava et al. 2014). Such growth inhibition of root length and biomass of Arabidopsis thaliana by Cyandermella asteris compounds was observed in close-distance cocultivations on nonseparated Petri dishes (Fig. 2). The concentration of the fungal compounds (e.g., such phytotoxins or astins) was presumably highest close to $C$. asteris and decreased at longer distances from $C$. asteris. In addition, A. thaliana plants accumulated higher anthocyanin levels when they grew closer to $C$. asteris, whereas this effect was not observed in cocultivations in bipartite Petri dishes (Supplementary Fig. S8; Table 1), indicating the presence of at least one toxic compound in the closer surroundings of the fungal colonies. The biosynthesis of anthocyanins is usually upregulated by environmental stresses such as drought, extreme temperatures, radiation and strong light, nutrient deficiencies, wounding, bacterial and fungal infections, or attack by

B
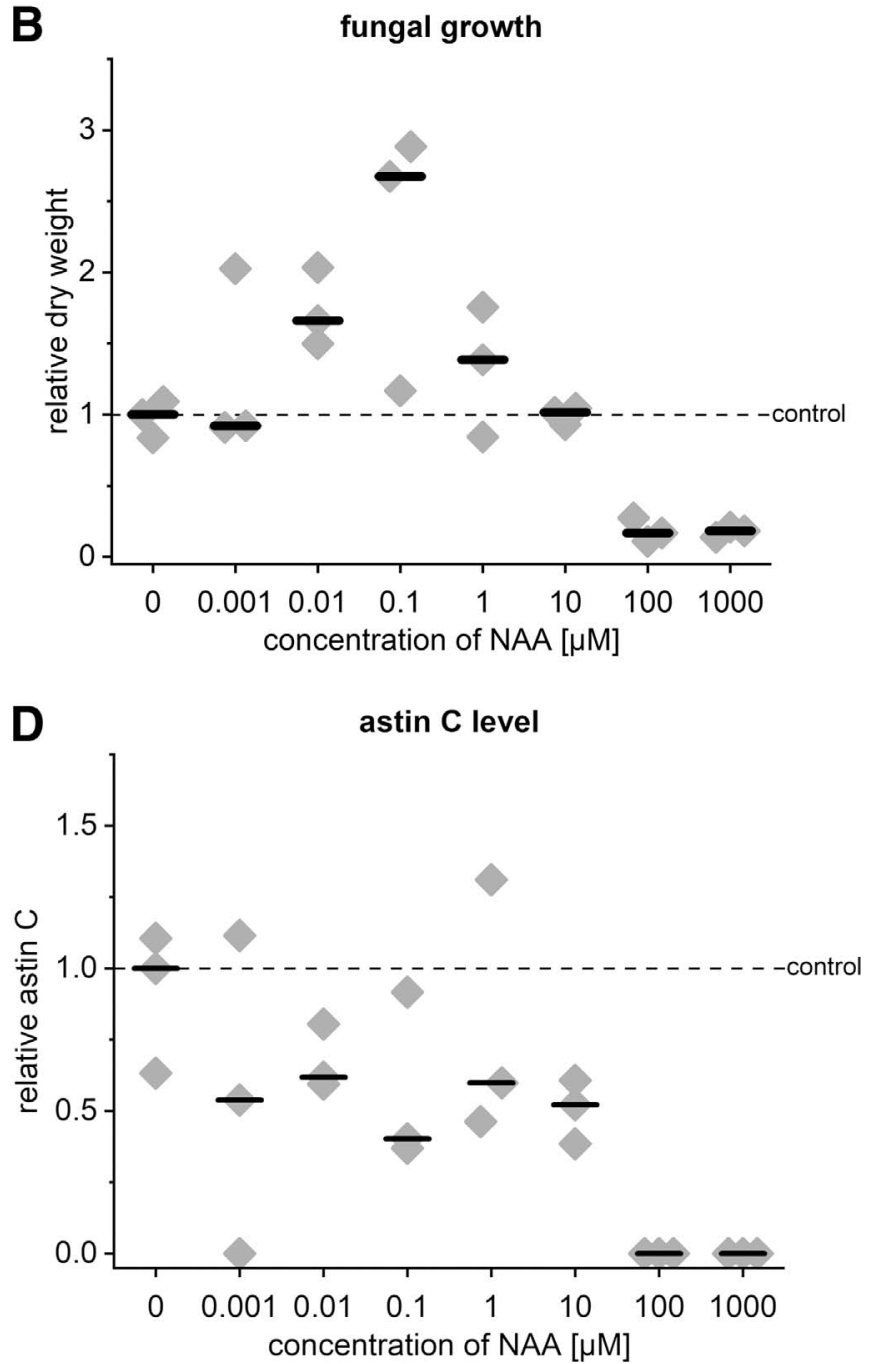

Fig. 9. Relative fungal growth and astin $\mathrm{C}$ production of Cyanodermella asteris in the presence of two different auxins. C. asteris was grown in liquid culture (malt extract without sucrose [MEAlow] including auxins) at $23^{\circ} \mathrm{C}$ in the dark for 1 week. Fungal growth in the presence of A, indole-3-acetic acid (IAA) and B, 1-naphthaleneacetic acid (NAA). Astin C production of $C$. asteris in the presence of $\mathbf{C}$, IAA and D, NAA. Relative values are related to the control without any additives $(0 \mu \mathrm{M})$. Sample size $=3$; bar indicates median. 
herbivores (Chalker-Scott 1999; McClure 1979). Because C. asteris is not a natural endophyte of A. thaliana, it may not be adapted to the fungus and its secreted metabolites. An incompatibility between $A$. thaliana and $C$. asteris was most likely indicated by the strong pigmentation of the plant because the anthocyanin content was higher when A. thaliana and $C$. asteris grew within a close distance (Supplementary Fig. S8). In addition to unknown fungi, natural endophytes can also lead to chlorosis or necrosis in A. thaliana leaves, especially under stressful conditions such as in vitro cultivation (Junker et al. 2012). Furthermore, plants can also react with direct defense responses to natural endophytes (Mandyam et al. 2013).

It was shown here that JA and SA as well as ethylene are typical stress hormones involved in the pathogen response (Broekaert et al. 2006; Halim et al. 2006) but were not involved in the development of the altered growth phenotype of A. thaliana. Mutants in the SA signaling (nprl-1) (Cao et al. 1997) and biosynthetic pathway (sid2-1) (Wildermuth et al. 2001) as well as transgenic plants overexpressing bacterial SA hydroxylase (NahG) (Friedrich et al. 1995) showed the same phenotype as the wild type after inoculation with $C$. asteris (Supplementary Fig. S9). Similarly, the jarl-1 mutant, which is defective in the
JA response (Staswick et al. 1992), and the etrl-1 mutant, which cannot perceive the ethylene signal (Gamble et al. 2002), showed the same altered growth phenotype; namely, shorter roots and more laterals. If other growth-promoting phytohormones such as cytokinins or gibberellins are involved in the interaction between A. thaliana and C. asteris, is a question currently under investigation.

When the plant and the fungus were separated by a barrier, only VOCs and $\mathrm{CO}_{2}$ are biologically active; however, fewer stress symptoms were developed (root growth and anthocyanin content). It appears that atmospheric $\mathrm{CO}_{2}$ (released by the fungus in the in vitro coculture system) primarily reduces plant stress in closed systems. If the cocultivation took place on bipartite Petri dishes with MaiMed tape (to mimic an open system), then the stress symptoms were no longer visible in the interaction of $A$. thaliana and $C$. asteris. This was also reported by Kai and Piechulla (2009), who analyzed the influence of VOCs produced by $B$. subtilis on A. thaliana but did not observe growth promotions as Ryu et al. (2003) had. However, the experimental settings were different. Kai and Piechulla (2009) used an open system where the air could circulate with the environment, whereas Ryu et al. (2003) used a closed system where the
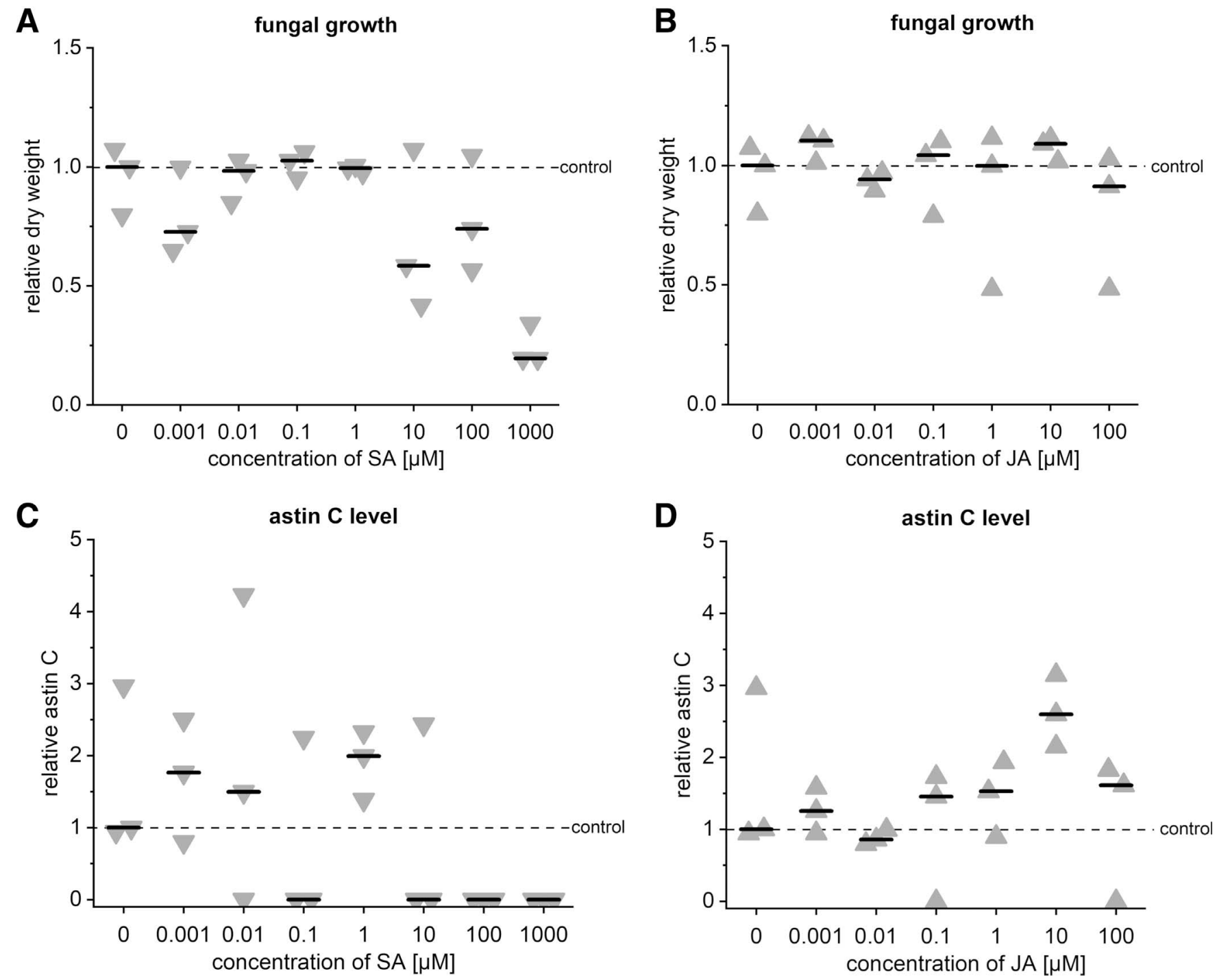

Fig. 10. Fungal growth and astin production of Cyanodermella asteris under different stress hormones. C. asteris was grown in a liquid culture (malt extract without sucrose [MEAlow] including salicylic acid [SA] or jasmonic acid [JA]) at $23^{\circ} \mathrm{C}$ in the dark for 1 week. Fungal growth in the presence of $\mathbf{A}, \mathrm{SA}$ and $\mathbf{B}, \mathrm{JA}$. Astin $\mathrm{C}$ production of $C$. asteris in the presence of $\mathbf{C}, \mathrm{SA}$ and $\mathbf{D}$, JA. Relative values are related to the control without any additives $(0 \mu \mathrm{M})$. Sample size $=3$; bar indicates median. 
concentrations of VOCs and $\mathrm{CO}_{2}$ are presumably much higher than in the natural environment.

Due to the low concentrations of VOCs, the structure of the bioactive VOCs could not be determined thus far. In contrast, the dichlorinated astin $\mathrm{C}$ produced by $C$. asteris induced plant development of $A$. thaliana (Supplementary Fig. S12). The fungal astin $\mathrm{C}$ was less biologically active than astin $\mathrm{A}$, which is only produced by the host plant Aster tataricus. The latter is presumably synthesized in plants by hydroxylation from the fungal astin C (Schafhauser et al. 2019) and might play a major role in the plant-endophyte interaction. It is known that compounds from endophytes can induce plant growth in the nonhost plant Arabidopsis thaliana. For example, the class of strobilurines that were primarily isolated from the fungal genus Strobilurus (Anke et al. 1977), also found in other fungi (Schramm et al. 1978), are used as fungicides in agriculture, and treated $A$. thaliana plants exhibited growth promotions due to an increased expression of sugar transport genes (Van Dingenen et al. 2017). In addition to the direct way to positively influence the plant host growth, there are also indirect ways to benefit the plant by endophytes; for example, release of siderophores to improve nutrition uptake of iron (Chhabra and Dowling 2017) or release of acid phosphatases to enable uptake of insoluble phosphates (Gill et al. 2016; Resende et al. 2014; Singh et al. 2003).

A third group of soluble compounds, which play an important role in plant-endophyte interactions, are plant hormones. Plant hormones are produced (Contreras-Cornejo et al. 2009; Sirrenberg et al. 2007) or concentrations are modulated (Shahzad et al. 2017) inside the plant by endophytes. B. amyloliquefaciens RWL-1, for example, induced the amino acid biosynthesis and the biosynthesis of the stress hormone JA in rice under salinity stress (Shahzad et al. 2017). Plant hormones produced by endophytes are auxinsSerendipita indica (Sirrenberg et al. 2007), T. virens (ContrerasCornejo et al. 2009), or Serratia marcescens (Shi et al. 2010) —and cytokinins - Serendipita indica (Vadassery et al. 2008) but also gibberellic acid (Bilal et al. 2018) as well as other hormones (Morrison et al. 2015) that play a role in the interaction between plant and endophyte. Here, we showed that plant hormones such as auxins and JA influence the fungal growth and astin production of $C$. asteris (Figs. 9 and 10). Because C. asteris synthesizes the natural auxin IAA de novo in in vitro cultures (Jahn et al. 2021), most likely to influence the plant, it is more interesting that the growth of $C$. asteris can be influenced by the more stable auxin NAA in in vitro cultures (Fig. 9). Saccharomyces cerevisiae differentiates from a yeast into a filamentous form in the presence of IAA (Prusty et al. 2004), involving an IAA-dependent gene expression in the yeast. These IAA-dependent gene expressions in microorganisms together with the fungal IAA biosynthesis and the auxin-dependent fungal growth in $C$. asteris strengthen the hypothesis that IAA is not only a plant but also a microbial signal.

Particularly interesting is that $C$. asteris produced most of the astin $\mathrm{C}$ in close contact with $A$. thaliana in closed experimental systems (Fig. 8). Because $C$. asteris lives inside its host plant Astertataricus, it is essential for the fungus to react to plant signals, especially those that are in its direct surroundings in the plant. For many endophytes, it is even essential for their survival to perceive plant signals, because they may die or become saprophytes when the plant dies. For example, Serendipita indica induces gene expression of hydrolytic enzymes and nutrient transporters in barley as soon as nitrogen deficiency occurs in dying plant cells. This switch from endophytic to saprophytic life style is associated with a morphological change to thinner hyphae (Lahrmann et al. 2013). Understanding the communication between $C$. asteris and A. thaliana will not only contribute to a better understanding of the $C$. asteris-Aster tataricus relationship but also add to our general understanding of plant-endophyte interactions.
What can we learn from our results on the interaction with the endophytic fungus $C$. asteris obtained for the nonnatural host Arabidopsis thaliana in the host Aster tataricus? In the nonnatural host interaction, the responses ranged from a stress response to growth promotion, depending on which factor was analyzed (Fig. 11). Thus, we hypothesize that $C$. asteris could affect the overall performance of $A$. tataricus by the production of astin $\mathrm{C}$, which is most likely converted into astin A or B by the host (Schafhauser et al. 2019). Both astin A and C could enhance the growth of $A$. thaliana. Fungal IAA would additionally support plant growth and development. Plant signals such as IAA or SA could be signals to the fungus to sense the environmental stress and maybe activate the metabolism (e.g., astins) or gene expression of compounds, which support Aster tataricus. For the nonnatural host plant, it was shown that the plant hormones (auxins and stress hormones) influence growth and metabolism (astin $\mathrm{C}$ production) of the fungus $C$. asteris.

In addition to soluble compounds, volatiles play a major role in the interaction between plant and fungus. The presence of $\mathrm{CO}_{2}$ led to increased plant growth and earlier plant development in the coculture systems. However, the most challenging question remains to be answered: namely, whether and how much of the internally (from fungal respiration) released $\mathrm{CO}_{2}$ contributes to plant development and growth. This knowledge would be of particular interest, because the endophyte lives within the plant. Furthermore, as-yet-uninvestigated VOCs of the fungus seem to also induce plant growth. The identification of the putative volatile compounds of the fungus will be a subject of future research. In summary, we provided the first evidence of the role of volatile and nonvolatile metabolites involved in the fascinating Arabidopsis thaliana-Cyanodermella asteris (plant-endophyte) interaction.

\section{MATERIALS AND METHODS}

\section{Chemicals.}

If not otherwise indicated, all chemicals were obtained from Carl Roth GmbH \& Co. KG (Karlsruhe, Germany), Fisher Scientific GmbH (Schwerte, Germany), Merck KGaA (Darmstadt, Germany), or Duchefa Biochemie (Haarlem, The Netherlands). Astins were extracted from fungal cultures of $C$. asteris according to Schafhauser et al. (2019).

\section{Biological materials.}

The fungus $C$. asteris originated from our own culture (deposited under the accession number DSM 100826 at DSMZ, Braunschweig, Germany), isolated from Aster tataricus in 2013 (Jahn et al. 2017) and maintained on MEAlow medium.

The Arabidopsis thaliana ecotype Columbia (Col-0) used in the Petri dish assays was obtained from Nottingham Arabidopsis Stock Centre (NASC, Loughborough, U.K.). Mutants of $A$. thaliana were obtained from different sources: sid2-1 and nprl-1 from Regina Mencia (Universidad Nacional del Litoral, Santa Fe, Argentina), $N a h G$ from Sabine Rosahl (Institute of Plant Biochemistry Halle, Germany), and jarl-1 as well as etrl-1 from NASC (Nottingham, UK).

Seeds of sunflower (Helianthus annuus) and tomato (Solanum lycopersicum) were purchased from Hornbach (Bornheim, Germany). Seeds of rapeseed (Brassica napus 'Jumbo'), Chinese cabbage (Brassica rapa 'Cantonner Witkrop'), and maize (Zea mays) were obtained from the Sächsisches Landesamt für Umwelt, Landwirtschaft und Geologie (Nossen, Germany).

\section{Development of the cocultivation medium.}

Different media combinations were tested for the interaction Petri dish assay. A.thaliana was used as plant partner. Fungal (MEAlow: malt extract at $10 \mathrm{~g} /$ liter, D-glucose at $10 \mathrm{~g} / \mathrm{liter}$, 
peptone at $1 \mathrm{~g} /$ liter, and Hutner trace elements [Hutner et al. 1950] at $1 \mathrm{ml} /$ liter; pH 6.1) and plant (half- and full-strength MS [Murashige and Skoog 1962], including vitamins) media with or without sucrose were combined 1:1 (Supplementary Table S1). The medium with the best performance of both interaction partners was chosen for further experiments.

\section{Selection of a nonnatural host plant partner.}

Different plants were tested for their ability as plant partner in the cocultivation assays. In addition to sunflower as a member of the family Asteraceae, we also tested (model) plants such as A. thaliana, tomato, rapeseed, Chinese cabbage, and maize. All of these plants were tested on the cocultivation medium, which was best suited for A.thaliana and C.asteris. Depending on the size of the plants, we used either square Petri dishes (12 by $12 \mathrm{~cm}$ in size) or translucent plastic vessels ( 9 by 10 by $7 \mathrm{~cm}$ in size). Seeds were sterilized as follows: $2 \mathrm{~min}$ in $70 \%$ ethanol with $0.1 \%$ triton $\mathrm{X}-100$, followed by $10 \mathrm{~min}$ (sunflower, rapeseed, Chinese cabbage, and maize) or $25 \mathrm{~min}$ (tomato) in $1.2 \%$ sodium hypochlorite with $0.1 \%$ Triton X-100, and finished by washing seeds four to five times with sterile water. Sterile plants were inoculated with $10 \mu \mathrm{l}$ of $C$. asteris homogenate either below their roots $(4.5 \mathrm{~cm}$ away from the roots in square Petri dishes) or

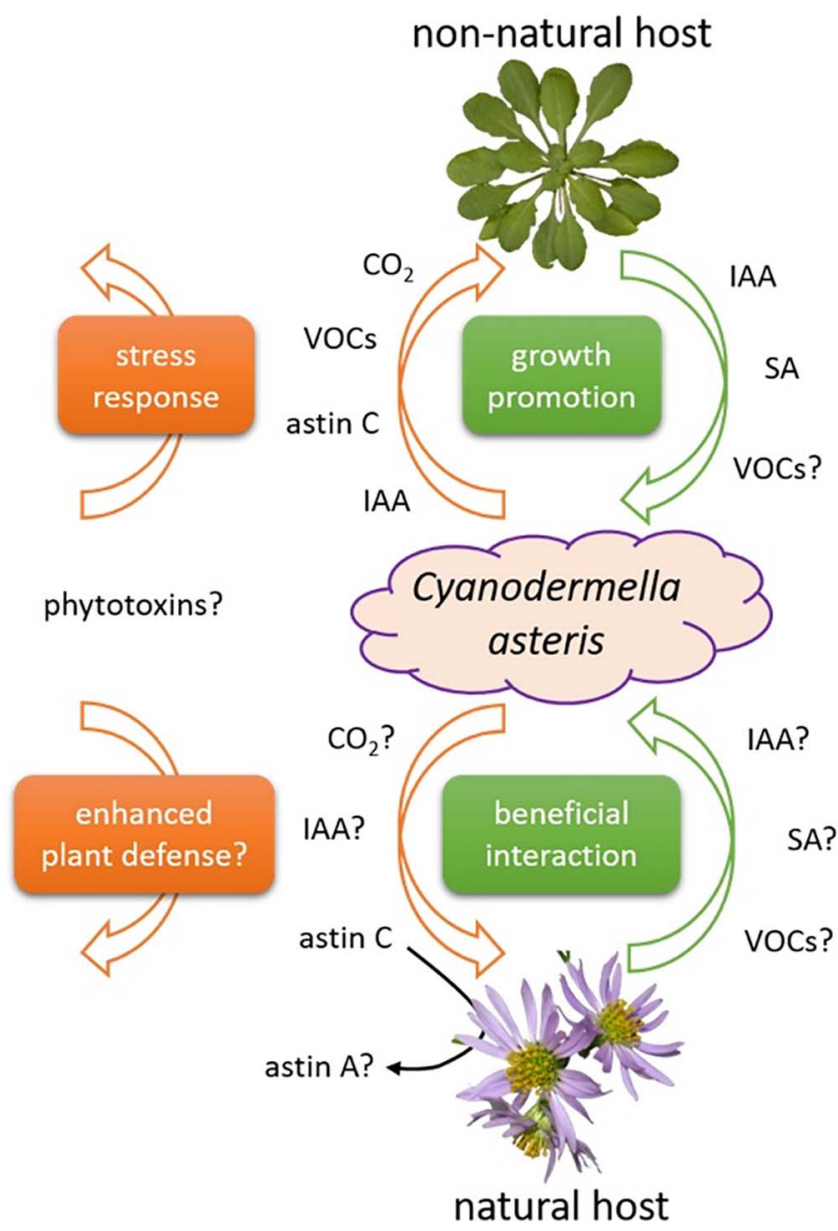

Fig. 11. Possible model of the interaction between the endophyte Cyanodermella asteris and the nonnatural host plant Arabidopsis thaliana as well as with its natural host plant Aster tataricus. C. asteris produces different volatile organic compounds (VOCs) and soluble compounds (including astin $\mathrm{C}$ and indole-3-acetic acid [IAA]) to support the nonnatural host plant Arabidopsis thaliana. In return, A. thaliana can use phytohormones (including IAA and salicylic acid [SA]) to induce fungal growth. The host plant Aster tataricus can additionally modify astin C into astin $\mathrm{A}$ or $\mathrm{B}$, which show a higher bioactivity. close to their roots (plastic vessels) within 1 week after sowing. The homogenate of C.asteris was from a 1-week-old culture, which was harvested by centrifugation $(5,000$ relative centrifugal force [rcf], $10 \mathrm{~min}$, room temperature). The pellet was resuspended in fresh MEAlow medium $(10 \mathrm{ml}$ per $1 \mathrm{ml}$ of pellet) and homogenated. The Petri dishes were sealed with Parafilm (Bemis, Neenah, WI, U.S.A.) and the translucent plastic vessels were closed with a lid. The plants were cultivated in the greenhouse under long-day conditions $\left(23^{\circ} \mathrm{C}\right.$ for $14 \mathrm{~h}$ under light, $18^{\circ} \mathrm{C}$ for $8 \mathrm{~h}$ in the dark; light intensities between 100 and $150 \mu \mathrm{mol} \mathrm{s}{ }^{-1} \mathrm{~m}^{-2}$ ) for three (Petri dishes) or four (plastic vessels) weeks. The plants best suited for in vitro assays according to their growth were chosen.

\section{Cocultivation Petri dish assays.}

All experimental setups were performed as shown in Supplementary Fig. S1. The experimental details for the different types of plates and sealings used will be separately described below.

Cocultivation in nonseparated Petri dishes. Prior to cocultivation experiments, $A$. thaliana seeds were soaked for $1 \mathrm{~min}$ in $70 \%$ ethanol including $0.1 \%$ Triton $\mathrm{X}-100$ followed by $1 \mathrm{~min}$ in $1.2 \%$ sodium hypochlorite including $0.1 \%$ Triton $\mathrm{X}-100$. The seeds were washed four times with sterile water to remove the chemical compounds. Seven sterile seeds were sown on nonseparated Petri dishes containing the chosen medium. After 1 week, the plates were inoculated with $C$. asteris (seven inocula) at different distances from the seedlings: $1.5,3$, and $4.5 \mathrm{~cm}$ (on round Petri dishes, $9 \mathrm{~cm}$ in diameter) as well as 1.5, 4.5, and $7.5 \mathrm{~cm}$ (on square Petri dishes, 12 by $12 \mathrm{~cm}$ in size) away from each seed. Each fungal inoculum contained the same amount of conidia (each inoculum with $10^{5}$ ). The conidia were harvested under sterile conditions from a 1-week-old culture of C. asteris (cultivation in MEAlow at $23^{\circ} \mathrm{C}, 130 \mathrm{rpm}$ in the dark) and collected after filtration over gauze (mesh size: $20 \mu \mathrm{m}$ ) by centrifugation $\left(5,000 \mathrm{rcf}, 4^{\circ} \mathrm{C}\right.$, and $\left.10 \mathrm{~min}\right)$. The conidia were resolved in a defined volume of MEAlow medium for application to A. thaliana plates. The Petri dishes were sealed with Parafilm or MaiMed tape (MaiMed GmbH, Neuenkirchen, Germany) and cultivated under long-day conditions $\left(23^{\circ} \mathrm{C}\right.$ for $14 \mathrm{~h}$ under light, $18^{\circ} \mathrm{C}$ for $8 \mathrm{~h}$ in the dark; light intensities between 90 and $120 \mu \mathrm{mol} \mathrm{s}{ }^{-1} \mathrm{~m}^{-2}$ ) in an upright position for 5 weeks (versatile plant growth chamber MLR-351H; Sanyo, Osaka, Japan). The phenotype of rosette growth, root length, and growth stages (Boyes et al. 2001) of A. thaliana was recorded weekly. After 35 days of cultivation, rosettes and roots were harvested separately, and fresh biomass and dry weight of both were determined per Petri dish. Each experiment on round Petri dishes $(9 \mathrm{~cm}$ in diameter) was performed with 10 Petri dishes. Experiments on square Petri dishes ( 12 by $12 \mathrm{~cm}$ in size) were done in triplicate and were not statistically analyzed. The results were reproducible between individual experiments. If the amount of fresh weight was below the detection limit of the scale, plant parts or organs of two or more Petri dishes were pooled for weighing. The freeze-dried rosettes were further used for chlorophyll and anthocyanin measurements.

Cocultivation of A. thaliana mutants in nonseparated Petri dishes. Different mutants and overexpressing lines of A. thaliana (jarl-1, etr1-1, sid2-1, npr1-1, and NahG) were inoculated with $10^{5}$ conidia of $C$. asteris in the same manner as the wild-type Col-0 (discussed above). The Petri dishes were sealed with MaiMed tape and cultivated under long-day conditions for 5 weeks.

Cocultivation in nonseparated Petri dishes with autoclaved fungus. A. thaliana Col-0 was grown as described above but supplemented with dead spores or dead hyphae. The spores or hyphae of $C$. asteris were propagated as described above for living materials but, after resuspension in MEAlow medium, they were autoclaved for $20 \mathrm{~min}$ at $121^{\circ} \mathrm{C}$ and $1.96 \mathrm{bar}$. The resulting 
dead spores $\left(5 \mu \mathrm{l}\right.$ with $10^{5}$ conidia) or dead hyphae $(5 \mu \mathrm{l}$ of homogenate) were applied 7 days after sowing $1.5 \mathrm{~cm}$ as well as $4.5 \mathrm{~cm}$ below the seedlings of $A$. thaliana.

Cocultivation on bipartite Petri dishes A. thaliana and C. asteris. Bipartite Petri dishes containing the cocultivation medium were set up as follows: 1 week after sowing three A. thaliana seeds, C. asteris was inoculated in the second compartment with three inocula of each $10^{5}$ conidia and subsequently spread uniformly over the compartment. Conidia were obtained from a 1-week-old C. asteris culture (discussed above). The bipartite Petri dishes were sealed either with Parafilm (20 Petri dishes) or MaiMed tape (19 Petri dishes) and cultivated as above. The plants were monitored weekly and harvested after 35 days (discussed above).

Cocultivation on tripartite Petri dishes A. thaliana and C. asteris. Tripartite Petri dishes containing the cocultivation medium were set up as follows. One compartment contained five $A$. thaliana seeds and, after 1 week, four inocula of each $10^{5}$ conidia of $C$. asteris were applied to the second compartment. The third compartment contained $7 \mathrm{ml}$ of $0.1 \mathrm{M} \mathrm{Ba}(\mathrm{OH})_{2}$ for the absorption of $\mathrm{CO}_{2}$ (Kai and Piechulla 2009). The Petri dishes were sealed with Parafilm (12 Petri dishes) and placed horizontally under long-day conditions (discussed above). The same parameters were determined as above. In addition, the amount of $\mathrm{BaCO}_{3}$ was determined after harvest (35 days after sowing). The dry weight of $\mathrm{BaCO}_{3}$ was measured after filtration (folded filters, $\varnothing 150 \mathrm{~mm}, 84 \mathrm{~g} / \mathrm{m}^{2}$, grade 1288; Sartorius Stedim Biotech, Göttingen, Germany) and drying at $60^{\circ} \mathrm{C}$ (incubator Heraeus Function Line T12; Heraeus, Hanau, Germany) for 1 week.

Permeability of sealing. The $\mathrm{CO}_{2}$ permeability of the different sealings (Parafilm and MaiMed tape) was tested both in the compartment with C.asteris and in that with A.thaliana. One compartment contained the cocultivation medium, the other $10 \mathrm{ml}$ of $0.1 \mathrm{M} \mathrm{Ba}(\mathrm{OH})_{2}$ for complexing $\mathrm{CO}_{2}$. The permeability was tested with $C$. asteris as well as A. thaliana. Three inocula of C. asteris $\left(10^{5}\right.$ conidia each inocula) or three seeds of $A$. thaliana were placed on the agar. The control Petri dishes contained no organism. Petri dishes of all three conditions were sealed in three different ways: no sealing (open system) or MaiMed tape or Parafilm (closed system). The amount of $\mathrm{BaCO}_{3}$ was determined after 28 days. At this time, Petri dishes without any sealing were dried out and could not be used as control. The results of the remaining Petri dishes were statistically analyzed using a Welch analysis of variance (ANOVA) with treatment (organism and sealing) as between-subject factors. A posthoc test was performed to show the differences between the groups and the effect size partial $\eta$ square $\left(\eta_{\mathrm{p}}{ }^{2}\right)$ was determined.

\section{Extraction and detection of anthocyanins and chlorophylls from plants.}

Freeze-dried plant material (rosettes) was ground with pestle and mortar and extracted with either $3 \mathrm{ml}$ of alkaline methanol ( $\mathrm{pH}$ 8.5) for chlorophylls or $3 \mathrm{ml}$ of acid methanol $(\mathrm{pH} 1)$ for anthocyanins. Depending on the biomass, plants of one or more Petri dishes were combined and extracted together. The extract was incubated for $5 \mathrm{~min}$ prior to centrifugation $\left(12,000 \mathrm{rcf}, 4^{\circ} \mathrm{C}\right.$, $10 \mathrm{~min})$. The supernatant was directly used in photometric measurements. The absorbance was measured at 645 and $663 \mathrm{~nm}$ for chlorophylls and at 530 and $657 \mathrm{~nm}$ for anthocyanins.

The chlorophyll concentration was determined according to formula I to III (Arnon 1949):

$$
\begin{gathered}
C(\text { chlorophyll } a[\mathrm{mg} / \text { liter }])=12.7 \times A_{663 \mathrm{~nm}}-2.69 \times A_{645 \mathrm{~nm}} \\
C(\text { chlorophyll } b[\mathrm{mg} / \text { liter }])=22.9 \times A_{645 \mathrm{~nm}}-4.68 \times A_{663 \mathrm{~nm}} \\
C(\text { chlorophyll total }[\mathrm{mg} / \text { liter }])= \\
C(\text { chlorophyll } a)+C(\text { chlorophyll b })
\end{gathered}
$$

The chlorophyll concentrations were calculated according to the extraction volume and the fresh weight (FW) of leaves (formula IV):

$$
\begin{gathered}
c(\text { chlorophyll }[\mu \mathrm{g} / \mathrm{g}])=\{c(\text { chlorophyll })[\mathrm{mg} / \text { liter }]) \times \\
1,000[\mu \mathrm{g} / \mathrm{mg}] \times \text { extraction volume }[\text { liter }]\} / F W[g]
\end{gathered}
$$

The anthocyanin concentration was calculated according to Rabino and Mancinelli (1986), which uses a correction factor of 0.25 . The correction factor compensates the absorption of chlorophyll and its degradation products at $530 \mathrm{~nm}$ (Mancinelli and Schwartz 1984) (formula V).

$$
\text { anthocyanin absorption }=A_{530 \mathrm{~nm}}-0.25 \times A_{657 \mathrm{~nm}}
$$

An anthocyanin equivalent was defined according to Teng et al. (2005) where the product of formula V with the extraction volume is one unit. The anthocyanin equivalents (unit) were related to the $\mathrm{FW}$ of leaves to compare the different treatments (formula VI).

$$
\begin{aligned}
& \text { anthocyanin content }[\text { unit } / g]= \\
& \quad(\text { anthocyanin absorption } \times \text { extraction volume }) / F W[g]
\end{aligned}
$$

\section{Extraction and detection of astins from Petri dishes.}

Freeze-dried medium of Petri dishes was ground with pestle and mortar and extracted with $100 \%$ methanol for 30 min on a shaker. Afterward, the solution was filtered to remove the solid particles from the solution. The methanol was vacuum dried in a Multivapor P-12 (Büchi, Essen, Germany). The resulting dried components were resolved in 50\% methanol and measured by highperformance liquid chromatography (HPLC) mass spectrometry.

The samples were analyzed on a Shimadzu HPLC system (Shimadzu, Canby, OR, U.S.A.) with a ProntoSIL 120-5 C18 AQ $250 \times 3 \mathrm{~mm}$ (Knauer Wissenschaftliche Geräte $\mathrm{GmbH}$, Berlin, Germany) and a Chromolith SpeedROD RP-18e 50 to $4.6 \mathrm{~mm}$ as precolumn. The astins were separated with an isocratic system (flow $0.7 \mathrm{ml} / \mathrm{min}$ ) of $40 \%$ acetonitrile plus $0.06 \%$ formic acid and $60 \%$ water plus $0.1 \%$ formic acid. The HPLC was coupled to an AB Sciex API 5000 Turbo-Ion-Spray triple quadrupole tandem mass spectrometer equipped with Electrospray Ionization (ESI) Source (AB Sciex, Foster City, CA, U.S.A.). The system was controlled by AB Sciex Analyst software (version 1.5.1). Due to a lack of internal standards for astins, only an estimation of the astin amount by area units was possible. A standard curve ( 0.1 to $10 \mathrm{ng} / \mathrm{ml}$ ) for astin $\mathrm{C}$ was done to link the arbitrary unit to an astin $\mathrm{C}$ concentration.

\section{Growth of $A$. thaliana on different astin concentrations.}

A. thaliana was cultivated in Petri dishes containing MS medium (MS including vitamins at $4.4 \mathrm{~g} /$ liter, sucrose at $10 \mathrm{~g} / \mathrm{liter}$, and phytoagar at $10 \mathrm{~g} /$ liter; $\mathrm{pH} 5.8 \pm 0.1$ ) (Murashige and Skoog 1962), including different concentrations of astins A, C, (both dichlorinated), or $\mathrm{G}$ (nonchlorinated). Seven plants were sown per Petri dish (10 Petri dishes per treatment). The growth of plants was documented weekly as in the cocultivation system (root length and growth stage). After 35 days of cultivation under long-day conditions, the plants were harvested and the biomass was determined.

Plate medians of root length and leaf biomass of $A$. thaliana treated with different astins were statistically analyzed using a one-way ANOVA (equal sample sizes and variance homogeneity) or Welch-ANOVA (no variance homogeneity), with treatment group (concentration) included as an eight-level (astins A and C) or seven-level (astin G) within-subject factor $(n=10)$. Posthoc tests were performed to specify the differences. The growth stages 35 days after cultivation were analyzed using a Kruskal-Wallis ANOVA ( $n=62$ to 70), with treatment group (concentration) as an eight-level (astins A and C) or seven-level (astin G) withinsubject factor. A posthoc test (Dunn's) was performed to show the 
differences. Leaf and root biomass were statistically analyzed using a one-way ANOVA or Welch-ANOVA, with concentration included as a within-subjects factor $(n \geq 10)$ or with a KruskalWallis ANOVA $(n<10)$. Posthoc tests were performed to show the differences between the concentrations. Effect sizes were calculated for significant differences using $\eta^{2}$ (Lenhard and Lenhard 2016) or $\eta_{\mathrm{p}}{ }^{2}$.

\section{Growth and astin production of $C$. asteris with phytohormones.}

C. asteris was cultivated in liquid MEAlow medium (Schafhauser et al. 2019) with different phytohormones (auxins: NAA and IAA; stress hormones: SA and JA) at different concentrations $(10 \mathrm{pM}$ to $1 \mu \mathrm{M}$, dissolved and diluted in methanol or water). IAA is very sensitive to light, temperature, and salty conditions and not stable enough in medium for a long-term experiment (Dunlap et al. 1986; Nissen and Sutter 1990). Therefore, the natural auxin IAA and the synthetic auxin NAA were tested.

Liquid medium ( $40 \mathrm{ml}$ ) was inoculated with $200 \mu$ of homogenate of $C$. asteris (discussed above). The fungus was cultivated for 1 week on a shaker at $23^{\circ} \mathrm{C}$ and $130 \mathrm{rpm}$ in the dark. The liquid medium was centrifuged $\left(5,000 \mathrm{rcf}, 4^{\circ} \mathrm{C}, 10 \mathrm{~min}\right)$ to remove the mycelium. The supernatant (medium) was extracted with the same volume of ethyl acetate for $30 \mathrm{~min}$ on a rotary shaker. The phases were separated by centrifugation $\left(5,000 \mathrm{rcf}, 4^{\circ} \mathrm{C}, 10 \mathrm{~min}\right)$ and the upper phase was vacuum evaporated. The resulting sediments were resolved in $1 \mathrm{ml}$ of $50 \%$ methanol and measured by HPLC mass spectrometry (discussed above). The pellet (mycelium) was freeze dried and the fungal biomass was determined.

\section{Statistical analyses.}

Statistical analyses were generally carried out using OriginPro 2019 version 9.6.0.172 (OriginLab Corporation, Northampton, MA, U.S.A.). Statistically significant differences are given in the figures and respective legends as well as in Supplementary Tables S2 to S10.

For analyses of growth parameters, it has to be considered that the plants within one Petri dish interact with each other through the Petri dish atmosphere. They influence the growth of other plants in the Petri dish. To show a nonindependence of plant interactions statistically, medians of root length, which were measured for each plant, were calculated for each Petri dish separately and used for further statistical analyses. All other growth parameters did not show such a dependence, because they were determined per Petri dish and not per plant. The growth parameters root length, leaf and root biomass, and chlorophyll and anthocyanin content with two groups (control and + $C$. asteris) were statistically analyzed using an unpaired $t$ test $(n \geq 10)$ or a Mann-Whitney $U$ test $(n<10)$. Growth parameters with more than two groups (control; $1.5,3.0$, or $4.5 \mathrm{~cm}$; or control \pm C. asteris $\pm \mathrm{Ba}(\mathrm{OH})_{2}$ ) were analyzed using a Welch-ANOVA (to allow for a different sample size $[n]$ ) with treatment as a between-subjects factor $(n \geq 10)$ or a Kruskal-Wallis test with treatment as a within-subjects factor for smaller sample sizes $(n<10)$. The growth stages were analyzed using the nonparametric Mann-Whitney $U$ test (two groups: control and $+C$. asteris) or the Kruskal-Wallis test (more than two groups: control; 1.5 , 3.0 , or $4.5 \mathrm{~cm}$; or control \pm C. asteris $\left.\pm \mathrm{Ba}(\mathrm{OH})_{2}\right)$. Variance homogeneity was checked with a Levene's test. Effect sizes were calculated using Cohen's $d$ (Lenhard and Lenhard 2016) or $\eta_{\mathrm{p}}{ }^{2}$.

\section{ACKNOWLEDGMENTS}

The experiments would not have been possible without the technical assistance of F. Benade and S. Rößler, Technische Universität Dresden.

\section{LITERATURE CITED}

Abdelaziz, M. E., Kim, D., Ali, S., Fedoroff, N. V., and Al-Babili, S. 2017. The endophytic fungus Piriformospora indica enhances Arabidopsis thaliana growth and modulates $\mathrm{Na}^{+} / \mathrm{K}^{+}$homeostasis under salt stress conditions. Plant Sci. 263:107-115.

Ali, S., Charles, T. C., and Glick, B. R. 2017. Endophytic phytohormones and their role in plant growth promotion. Pages 89-105 in: Functional Importance of the Plant Microbiome. S. L. Doty, ed. Springer International Publishing, Cham, Switzerland.

Anke, T., Oberwinkler, F., Steglich, W., and Schramm, G. 1977. The strobilurins-New antifungal antibiotics from the basidiomycete Strobilurus tenacellus. J. Antibiot. (Tokyo) 30:806-810.

Arnon, D. I. 1949. Copper enzymes in isolated chloroplasts. Polyphenoloxidase in Beta vulgaris. Plant Physiol. 24:1-15.

Bilal, L., Asaf, S., Hamayun, M., Gul, H., Iqbal, A., Ullah, I., Lee, I.-J., and Hussain, A. 2018. Plant growth promoting endophytic fungi Aspergillus fumigatus TS1 and Fusarium proliferatum BRL1 produce gibberellins and regulates plant endogenous hormones. Symbiosis 76:117-127.

Boyes, D. C., Zayed, A. M., Ascenzi, R., McCaskill, A. J., Hoffman, N. E., Davis, K. R., and Görlach, J. 2001. Growth stage-based phenotypic analysis of Arabidopsis: A model for high throughput functional genomics in plants. Plant Cell 13:1499-1510.

Broekaert, W. F., Delauré, S. L., De Bolle, M. F. C., and Cammue, B. P. A. 2006. The role of ethylene in host-pathogen interactions Annu. Rev. Phytopathol. 44:393-416.

Cao, H., Glazebrook, J., Clarke, J. D., Volko, S., and Dong, X. 1997. The Arabidopsis NPR1 gene that controls systemic acquired resistance encodes a novel protein containing ankyrin repeats. Cell 88:57-63.

Casarrubia, S., Sapienza, S., Fritz, H., Daghino, S., Rosenkranz, M., Schnitzler, J.-P., Martin, F., Perotto, S., and Martino, E. 2016. Ecologically different fungi affect Arabidopsis development: Contribution of soluble and volatile compounds. PLoS One 11:e0168236.

Chalker-Scott, L. 1999. Environmental significance of anthocyanins in plant stress responses. Photochem. Photobiol. 70:1-9.

Chhabra, S., and Dowling, D. N. 2017. Endophyte-promoted nutrient acquisition: Phosphorus and iron. Pages 21-42 in: Functional Importance of the Plant Microbiome. S. L. Doty, ed. Springer International Publishing, Cham, Switzerland.

Contesto, C., Milesi, S., Mantelin, S., Zancarini, A., Desbrosses, G., Varoquaux, F., Bellini, C., Kowalczyk, M., and Touraine, B. 2010. The auxin-signaling pathway is required for the lateral root response of Arabidopsis to the rhizobacterium Phyllobacterium brassicacearum. Planta 232:1455-1470.

Contreras-Cornejo, H. A., Macías-Rodríguez, L., Cortés-Penagos, C., and López-Bucio, J. 2009. Trichoderma virens, a plant beneficial fungus, enhances biomass production and promotes lateral root growth through an auxin-dependent mechanism in Arabidopsis. Plant Physiol. 149: 1579-1592.

Dobbelaere, S., Croonenborghs, A., Thys, A., Vande Broek, A., and Vanderleyden, J. 1999. Phytostimulatory effect of Azospirillum brasilense wild type and mutant strains altered in IAA production on wheat. Plant Soil 212:153-162.

Dovana, F., Mucciarelli, M., Mascarello, M., and Fusconi, A. 2015. In vitro morphogenesis of Arabidopsis to search for novel endophytic fungi modulating plant growth. PLoS One 10:e0143353.

Dunlap, J. R., Kresovich, S., and McGee, R. E. 1986. The effect of salt concentration on auxin stability in culture media. Plant Physiol. 81:934-936.

Friedrich, L., Vernooij, B., Gaffney, T., Morse, A., and Ryals, J. 1995. Characterization of tobacco plants expressing a bacterial salicylate hydroxylase gene. Plant Mol. Biol. 29:959-968.

Gamble, R. L., Qu, X., and Schaller, G. E. 2002. Mutational analysis of the ethylene receptor ETR1. Role of the histidine kinase domain in dominant ethylene insensitivity. Plant Physiol. 128:1428-1438.

Gill, S. S., Gill, R., Trivedi, D. K., Anjum, N. A., Sharma, K. K., Ansari, M. W., Ansari, A. A., Johri, A. K., Prasad, R., Pereira, E., Varma, A., and Tuteja, N. 2016. Piriformospora indica: Potential and significance in plant stress tolerance. Front. Microbiol. 7:332.

Gonzalez, M. C., Anaya, A. L., Glenn, A. E., Saucedo-Garcia, A., Macias-Rubalcava, M. L., and Hanlin, R. T. 2007. A new endophytic ascomycete from El Eden Ecological Reserve, Quintana Roo, Mexico. Mycotaxon 101:251.

González-Pérez, E., Ortega-Amaro, M. A., Salazar-Badillo, F. B., Bautista, E., Douterlungne, D., and Jiménez-Bremont, J. F. 2018. The ArabidopsisTrichoderma interaction reveals that the fungal growth medium is an important factor in plant growth induction. Sci. Rep. 8:16427. 
Halim, V. A., Vess, A., Scheel, D., and Rosahl, S. 2006. The role of salicylic acid and jasmonic acid in pathogen defence. Plant Biol. 8:307-313.

Hutner, S. H., Provasoli, L., Schatz, A., and Haskins, C. P. 1950. Some approaches to the study of the role of metals in the metabolism of microorganisms. Proc. Am. Philos. Soc. 94:152-170.

Itokawa, H., Morita, H., Nagashima, S., and Takeya, K. 1994. Cyclic peptides from higher plants. Part 8. Three novel cyclic pentapeptides, astins F, G and H from Aster tataricus. Heterocycles 38:2247-2252.

Jahn, L., Hofmann, U., and Ludwig-Müller, J. 2021. Indole-3-acetic acid is synthesized by the endophyte Cyanodermella asteris by a tryptophan-dependent and -independent way and mediates the interaction with a non-host plant. Int. J. Mol. Sci. 22:2651.

Jahn, L., Schafhauser, T., Pan, S., Weber, T., Wohlleben, W., Fewer, D., Sivonen, K., Flor, L., van Pée, K.-H., Caradec, T., Jacques, P., Huijbers, M. E., Berkel, W. H., and Ludwig-Müller, J. 2017. Cyanodermella asteris sp. nov. (Ostropales) from the inflorescence axis of Aster tataricus. Mycotaxon 132:107-123.

Jäschke, D., Dugassa-Gobena, D., Karlovsky, P., Vidal, S., and LudwigMüller, J. 2010. Suppression of clubroot (Plasmodiophora brassicae) development in Arabidopsis thaliana by the endophytic fungus Acremonium alternatum. Plant Pathol. 59:100-111.

Junker, C., Draeger, S., and Schulz, B. 2012. A fine line-Endophytes or pathogens in Arabidopsis thaliana. Fungal Ecol. 5:657-662.

Kai, M., and Piechulla, B. 2009. Plant growth promotion due to rhizobacterial volatiles-An effect of $\mathrm{CO}_{2}$ ? FEBS Lett. 583:3473-3477.

Kishimoto, K., Matsui, K., Ozawa, R., and Takabayashi, J. 2006a. Analysis of defensive responses activated by volatile allo-ocimene treatment in Arabidopsis thaliana. Phytochemistry 67:1520-1529.

Kishimoto, K., Matsui, K., Ozawa, R., and Takabayashi, J. 2006b. Components of $\mathrm{C}_{6}$-aldehyde-induced resistance in Arabidopsis thaliana against a necrotrophic fungal pathogen, Botrytis cinerea. Plant Sci. 170:715-723.

Kishimoto, K., Matsui, K., Ozawa, R., and Takabayashi, J. 2007. Volatile 1-octen-3-ol induces a defensive response in Arabidopsis thaliana. J. Gen. Plant Pathol. 73:35-37.

Kochar, M., Upadhyay, A., and Srivastava, S. 2011. Indole-3-acetic acid biosynthesis in the biocontrol strain Pseudomonas fluorescens Psd and plant growth regulation by hormone overexpression. Res. Microbiol. 162: 426-435.

Lahrmann, U., Ding, Y., Banhara, A., Rath, M., Hajirezaei, M. R. Döhlemann, S., von Wirén, N., Parniske, M., and Zuccaro, A. 2013. Hostrelated metabolic cues affect colonization strategies of a root endophyte. Proc. Natl. Acad. Sci. U.S.A. 110:13965-13970.

Lee, S., Behringer, G., Hung, R., and Bennett, J. 2019. Effects of fungal volatile organic compounds on Arabidopsis thaliana growth and gene expression. Fungal Ecol. 37:1-9.

Lenhard, W., and Lenhard, A. 2016. Calculation of effect sizes. Psychometrica, Bibergau, Germany. http://www.psychometrica.de/effect_size.htlm

López-Bucio, J., Campos-Cuevas, J. C., Hernández-Calderón, E., VelásquezBecerra, C., Farías-Rodríguez, R., Macías-Rodríguez, L. I., and ValenciaCantero, E. 2007. Bacillus megaterium rhizobacteria promote growth and alter root-system architecture through an auxin- and ethylene-independent signaling mechanism in Arabidopsis thaliana. Mol. Plant-Microbe Interact. 20:207-217.

Ludwig-Müller, J., Auer, S., Jülke, S., and Marschollek, S. 2017. Manipulation of auxin and cytokinin balance during the Plasmodiophora brassicae-Arabidopsis thaliana interaction. Pages 41-60 in: Methods in Molecular Biology. T. Dandekar and M. Naseem, eds. Springer, New York, NY, U.S.A

Macías-Rubalcava, M. L., Ruiz-Velasco Sobrino, M. E., Meléndez-González, C., and Hernández-Ortega, S. 2014. Naphthoquinone spiroketals and organic extracts from the endophytic fungus Edenia gomezpompae as potential herbicides. J. Agric. Food Chem. 62:3553-3562.

Madhu, M., and Hatfield, J. L. 2013. Dynamics of plant root growth under increased atmospheric carbon dioxide. Agron. J. 105:657-669.

MaiMed. 2011. Technical Data Sheet: MaiMed-pore. https://maimed. de/technical-data-sheets/?lang=en

Makino, A., and Mae, T. 1999. Photosynthesis and plant growth at elevated levels of $\mathrm{CO}_{2}$. Plant Cell Physiol. 40:999-1006.

Mancinelli, A. L., and Schwartz, O. M. 1984. The photoregulation of anthocyanin synthesis IX. The photosensitivity of the response in dark and light-grown tomato seedlings. Plant Cell Physiol. 25:93-105.

Mandyam, K. G., Roe, J., and Jumpponen, A. 2013. Arabidopsis thaliana model system reveals a continuum of responses to root endophyte colonization. Fungal Biol. 117:250-260.

McClure, J. W. 1979. The physiology of phenolic compounds in plants. Pages 525-556 in: Recent Advances in Phytochemistry. T. Swain, J. B. Harbone, and C. F. Van Sumere, eds. Springer, Boston, MA, U.S.A.
Mercier, J., and Manker, D. C. 2005. Biocontrol of soil-borne diseases and plant growth enhancement in greenhouse soilless mix by the volatile-producing fungus Muscodor albus. Crop Prot. 24:355-362.

Minerdi, D., Bossi, S., Maffei, M. E., Gullino, M. L., and Garibaldi, A. 2011. Fusarium oxysporum and its bacterial consortium promote lettuce growth and expansin A5 gene expression through microbial volatile organic compound (MVOC) emission. FEMS Microbiol. Ecol. 76:342-351.

Morath, S. U., Hung, R., and Bennett, J. W. 2012. Fungal volatile organic compounds: A review with emphasis on their biotechnological potential. Fungal Biol. Rev. 26:73-83.

Morita, H., Nagashima, S., Shirota, O., Takeya, K., and Itokawa, H. 1993a. Two novel monochlorinated cyclic pentapeptides, astins D and E from Aster tataricus. Chem. Lett. 22:1877-1880.

Morita, H., Nagashima, S., Takeya, K., and Itokawa, H. 1993b. Astins A and $\mathrm{B}$, antitumor cyclic pentapeptides from Aster tataricus. Chem. Pharm. Bull. (Tokyo) 41:992-993.

Morita, H., Nagashima, S., Takeya, K., and Itokawa, H. 1994. A novel cyclic pentapeptide with $\beta$-hydroxy- $\gamma$-chloroproline from Aster tataricus. Chem. Lett. 23:2009-2010.

Morita, H., Nagashima, S., Takeya, K., and Itokawa, H. 1995. Structure of a new peptide, astin J, from Aster tataricus. Chem. Pharm. Bull. (Tokyo) 43:271-273.

Morrison, E. N., Knowles, S., Hayward, A., Thorn, R. G., Saville, B. J., and Emery, R. J. N. 2015. Detection of phytohormones in temperate forest fungi predicts consistent abscisic acid production and a common pathway for cytokinin biosynthesis. Mycologia 107:245-257.

Murashige, T., and Skoog, F. 1962. A revised medium for rapid growth and bio assays with tobacco tissue cultures. Physiol. Plant. 15:473-497.

Nissen, S. J., and Sutter, E. G. 1990. Stability of IAA and IBA in nutrient medium to several tissue culture procedures. HortScience 25:800-802.

Ortíz-Castro, R., Valencia-Cantero, E., and López-Bucio, J. 2008. Plant growth promotion by Bacillus megaterium involves cytokinin signaling. Plant Signal. Behav. 3:263-265.

Peškan-Berghöfer, T., Shahollari, B., Giong, P. H., Hehl, S., Markert, C., Blanke, V., Kost, G., Varma, A., and Oelmüller, R. 2004. Association of Piriformospora indica with Arabidopsis thaliana roots represents a novel system to study beneficial plant-microbe interactions and involves early plant protein modifications in the endoplasmic reticulum and at the plasma membrane. Physiol. Plant. 122:465-477.

Piechulla, B., Lemfack, M. C., and Kai, M. 2017. Effects of discrete bioactive microbial volatiles on plants and fungi. Plant Cell Environ. 40:2042-2067.

Piechulla, B., and Schnitzler, J.-P. 2016. Circumvent $\mathrm{CO}_{2}$ effects in volatilebased microbe-plant interactions. Trends Plant Sci. 21:541-543.

Pons, S., Fournier, S., Chervin, C., Bécard, G., Rochange, S., Frei Dit Frey, N., and Puech Pagès, V. 2020. Phytohormone production by the arbuscular mycorrhizal fungus Rhizophagus irregularis. PLoS One 15 e0240886.

Proença, D. N., Schwab, S., Vidal, M. S., Baldani, J. I., Xavier, G. R., and Morais, P. V. 2019. The nematicide Serratia plymuthica M24T3 colonizes Arabidopsis thaliana, stimulates plant growth, and presents plant beneficial potential. Braz. J. Microbiol. 50:777-789.

Prusty, R., Grisafi, P., and Fink, G. R. 2004. The plant hormone indoleacetic acid induces invasive growth in Saccharomyces cerevisiae. Proc. Natl. Acad. Sci. U.S.A. 101:4153-4157.

Rabino, I., and Mancinelli, A. L. 1986. Light, temperature, and anthocyanin production. Plant Physiol. 81:922-924.

Reineke, G., Heinze, B., Schirawski, J., Buettner, H., Kahmann, R., and Basse, C. W. 2008. Indole-3-acetic acid (IAA) biosynthesis in the smut fungus Ustilago maydis and its relevance for increased IAA levels in infected tissue and host tumour formation. Mol. Plant Pathol. 9:339-355.

Resende, M. P., Jakoby, I. C. M. C., dos Santos, L. C. R., Soares, M. A., Pereira, F. D., Souchie, E. L., and Silva, F. G. 2014. Phosphate solubilization and phytohormone production by endophytic and rhizosphere Trichoderma isolates of guanandi (Calophyllum brasiliense Cambess). Afr. J. Microbiol. Res. 8:2616-2623.

Ryu, C.-M., Farag, M. A., Hu, C.-H., Reddy, M. S., Wei, H.-X., Paré, P. W., and Kloepper, J. W. 2003. Bacterial volatiles promote growth in Arabidopsis. Proc. Natl. Acad. Sci. U.S.A. 100:4927-4932.

Salas-Marina, M. A., Silva-Flores, M. A., Cervantes-Badillo, M. G., Rosales-Saavedra, M. T., Islas-Osuna, M. A., and Casas-Flores, S. 2011. The plant growth-promoting fungus Aspergillus ustus promotes growth and induces resistance against different lifestyle pathogens in Arabidopsis thaliana. J. Microbiol. Biotechnol. 21:686-696.

Schafhauser, T., Jahn, L., Kirchner, N., Kulik, A., Flor, L., Lang, A., Caradec, T., Fewer, D. P., Sivonen, K., van Berkel, W. J. H., Jacques, P., Weber, T., Gross, H., van Pée, K.-H., Wohlleben, W., and LudwigMüller, J. 2019. Antitumor astins originate from the fungal endophyte 
Cyanodermella asteris living within the medicinal plant Aster tataricus. Proc. Natl. Acad. Sci. U.S.A. 116:26909-26917.

Schramm, G., Steglich, W., Anke, T., and Oberwinkler, F. 1978. Antibiotika aus Basidiomyceten, III. Strobilurin A und B, antifungische Stoffwechselprodukte aus Strobilurus tenacellus. Chem. Ber. 111:2779-2784.

Schulz, B., and Boyle, C. 2005. The endophytic continuum. Mycol. Res. 109:661-686.

Shahzad, R., Khan, A. L., Bilal, S., Waqas, M., Kang, S.-M., and Lee, I.-J. 2017. Inoculation of abscisic acid-producing endophytic bacteria enhances salinity stress tolerance in Oryza sativa. Environ. Exp. Bot. 136:68-77.

Shao, Y., Ho, C. T., Chin, C. K., Poobrasert, O., Yang, S. W., and Cordell, G. A. 1997a. Asterlingulatosides C and D, cytotoxic triterpenoid saponins from Aster lingulatus. J. Nat. Prod. 60:743-746.

Shao, Y., Ho, C.-T., Chin, C.-K., Rosen, R. T., Hu, B., and Qin, G.-W. 1997b. Triterpenoid saponins from Aster lingulatus. Phytochemistry 44:337-340

Shi, C.-L., Park, H.-B., Lee, J. S., Ryu, S., and Ryu, C.-M. 2010. Inhibition of primary roots and stimulation of lateral root development in Arabidopsis thaliana by the rhizobacterium Serratia marcescens 90-166 is through both auxin-dependent and -independent signaling pathways. Mol. Cells 29:251-258.

Shirota, O., Morita, H., Takeya, K., Itokawa, H., and Iitaka, Y. 1997. Cytotoxic triterpenes from Aster tataricus. Nat. Med. 51:170-172.

Singh, A., Singh, A., Kumari, M., Rai, M. K., and Varma, A. 2003. Biotechnological importance of Piriformospora indica Verma et al.-A novel symbiotic mycorrhiza-like fungus: An overview. Indian J. Biotechnol. 2:65-75.

Singhal, U., Prasad, R., and Varma, A. 2017. Piriformospora indica (Serendipita indica): The novel symbiont. Pages 349-364 in: Mycorrhiza-Function, Diversity, State of the Art. Publishing. A. Varma, R. Prasad, and N. Tuteja, eds. Springer, Cham, Switzerland.

Sirrenberg, A., Göbel, C., Grond, S., Czempinski, N., Ratzinger, A., Karlovsky, P., Santos, P., Feussner, I., and Pawlowski, K. 2007. Piriformospora indica affects plant growth by auxin production. Physiol. Plant. 131:581-589.

Staswick, P. E., Su, W., and Howell, S. H. 1992. Methyl jasmonate inhibition of root growth and induction of a leaf protein are decreased in an Arabidopsis thaliana mutant. Proc. Natl. Acad. Sci. U.S.A. 89: 6837-6840.

Strobel, G. A., Dirkse, E., Sears, J., and Markworth, C. 2001. Volatile antimicrobials from Muscodor albus, a novel endophytic fungus. Microbiol. Read. 147:2943-2950.

Teng, S., Keurentjes, J., Bentsink, L., Koornneef, M., and Smeekens, S. 2005. Sucrose-specific induction of anthocyanin biosynthesis in Arabidopsis requires the MYB75/PAP1 gene. Plant Physiol. 139:1840-1852.

Vadassery, J., Ritter, C., Venus, Y., Camehl, I., Varma, A., Shahollari, B., Novák, O., Strnad, M., Ludwig-Müller, J., and Oelmüller, R. 2008. The role of auxins and cytokinins in the mutualistic interaction between Arabidopsis and Piriformospora indica. Mol. Plant-Microbe Interact. 21:1371-1383.

Van der Kooij, L. A. W., De Kok, L. J., and Stulen, I. 1999. Biomass production and carbohydrate content of Arabidopsis thaliana at atmospheric $\mathrm{CO}_{2}$ concentrations from 390 to $1680 \mathrm{\mu l}^{-1}$. Plant Biol. 1:482-486.

Van Dingenen, J., Antoniou, C., Filippou, P., Pollier, J., Gonzalez, N. Dhondt, S., Goossens, A., Fotopoulos, V., and Inzé, D. 2017. Strobilurins as growth-promoting compounds: How Stroby regulates Arabidopsis leaf growth. Plant Cell Environ. 40:1748-1760.

Verma, S., Varma, A., Rexer, K.-H., Hassel, A., Kost, G., Sarbhoy, A., Bisen, P., Bütehorn, B., and Franken, P. 1998. Piriformospora indica gen. et sp. nov., a new root-colonizing fungus. Mycologia 90:896-903.

Wildermuth, M. C., Dewdney, J., Wu, G., and Ausubel, F. M. 2001. Isochorismate synthase is required to synthesize salicylic acid for plant defence. Nature 414:562-565.

Worapong, J., Strobel, G., Ford, E. J., Li, J. Y., Baird, G., and Hess, W. M. 2001. Muscodor albus anam. gen. et sp. nov., an endophyte from Cinnamomum zeylanicum. Mycotaxon 79:67-79.

Xu, H.-M., Zeng, G.-Z., Zhou, W.-B., He, W.-J., and Tan, N.-H. 2013 Astins K-P, six new chlorinated cyclopentapeptides from Aster tataricus. Tetrahedron 69:7964-7969.

Xu, L., Wu, C., Oelmüller, R., and Zhang, W. 2018. Role of phytohormones in Piriformospora indica-induced growth promotion and stress tolerance in plants: More questions than answers. Front. Microbiol. 9:1646. 